TRANSACTIONS OF THE

AMERICAN MATHEMATICAL SOCIETY

Volume 362, Number 8, August 2010, Pages 4451-4479

S 0002-9947(10)05098-1

Article electronically published on March 17, 2010

\title{
THE RESOLVENT KERNEL FOR PCF SELF-SIMILAR FRACTALS
}

\author{
MARIUS IONESCU, ERIN P. J. PEARSE, LUKE G. ROGERS, HUO-JUN RUAN, \\ AND ROBERT S. STRICHARTZ
}

\begin{abstract}
For the Laplacian $\Delta$ defined on a p.c.f. self-similar fractal, we give an explicit formula for the resolvent kernel of the Laplacian with Dirichlet boundary conditions and also with Neumann boundary conditions. That is, we construct a symmetric function $G^{(\lambda)}$ which solves $(\lambda \mathbb{I}-\Delta)^{-1} f(x)=$ $\int G^{(\lambda)}(x, y) f(y) d \mu(y)$. The method is similar to Kigami's construction of the Green kernel and $G^{(\lambda)}$ is expressed as a sum of scaled and "translated" copies of a certain function $\psi^{(\lambda)}$ which may be considered as a fundamental solution of the resolvent equation. Examples of the explicit resolvent kernel formula are given for the unit interval, standard Sierpinski gasket, and the level-3 Sierpinski gasket $S G_{3}$.
\end{abstract}

\section{Contents}

1. Introduction

2. The resolvent kernel for the unit interval

3. The Dirichlet resolvent kernel for p.c.f. self-similar fractals

4. The Neumann resolvent kernel for p.c.f. self-similar fractals

5. Example: The Sierpinski gasket $S G$

4468

6. Example: $S G_{3}$, a variant of the Sierpinski gasket

\section{INTRODUCTION}

A theory of analysis on certain self-similar fractals is developed around the Laplace operator $\Delta$ in Kig01. In this paper, we consider the resolvent function

Received by the editors November 25, 2008 and, in revised form, April 20, 2009.

2010 Mathematics Subject Classification. Primary 28A80, 35P99, 47A75; Secondary 39A12, 39A70, 47B39.

Key words and phrases. Dirichlet form, graph energy, discrete potential theory, discrete Laplace operator, graph Laplacian, eigenvalue, resolvent formula, post-critically finite, self-similar, fractal.

The work of the second author was partially supported by the University of Iowa Department of Mathematics NSF VIGRE grant DMS-0602242.

The work of the fourth author was partially supported by grant NSFC 10601049 and by the Future Academic Star project of Zhejiang University.

The work of the fifth author was partially supported by NSF grant DMS 0652440.

(C)2010 American Mathematical Society Reverts to public domain 28 years from publication 
$(\lambda \mathbb{I}-\Delta)^{-1}$ and obtain a kernel for this function when the Laplacian is taken to have Dirichlet or Neumann boundary conditions. That is, we construct a symmetric function $G^{(\lambda)}$ which weakly solves $(\lambda \mathbb{I}-\Delta) G^{(\lambda)}(x, y)=\delta(x, y)$, meaning that

$$
\int G^{(\lambda)}(x, y) f(y) d \mu(y)=(\lambda \mathbb{I}-\Delta)^{-1} f(x) .
$$

For the case $\lambda=0$, this is just the Green function for $\Delta$. Consequently, it is not surprising that our construction is quite analogous to that of the Green function as carried out in [Kig01, §3.5]; see also [Str06, §2.6] for the case of the Sierpinski gasket (and the unit interval) worked out in detail, and Kig03.

We present our main results in 11.2 , just after the introduction of the necessary technical terms in \$1.1. It is the authors' hopes that the resolvent kernel will provide an alternate route to obtaining heat kernel estimates (see [FHK94, HK99]) in this setting, as well as other information about spectral operators of the form

$$
\xi(\Delta)=\int_{\Gamma} \xi(\lambda)(\lambda \mathbb{I}-\Delta)^{-1} d \lambda
$$

in the same manner as used by Seeley [See67, See69] for the Euclidean situation. Some initial results in this direction will appear in Rog08.

To explain the method of construction for the resolvent kernel, we carry out the procedure in the case of the unit interval in $\$ 2$, we believe this particular method has not previously appeared in the literature. In 43 , we show how the construction may be generalized to any post-critically finite self-similar fractal. In $\$$, we give the explicit formulas for the Sierpinski gasket and in $\$ 6$ we give the explicit formulas for a variant of the Sierpinski gasket which we call $S G_{3}$.

1.1. Background, notation, and fundamentals. We work in the context of post-critically finite (p.c.f.) self-similar fractals. The full and precise definition may be found in Kig01, Def. 1.3.13], but for the present context it suffices to think of such objects as fractals which may be approximated by a sequence of graphs, via an iterated function system (IFS). A more general setting is possible; cf. Kig03. We now make this more precise.

Definition 1.1. Let $\left\{F_{1}, F_{2}, \ldots, F_{J}\right\}$ be a collection of Lipschitz continuous functions on $\mathbb{R}^{d}$ with $0<\operatorname{Lip}\left(F_{j}\right)<1$ for each $j$. Let $X$ denote the attractor of this IFS, that is, $X$ is the unique nonempty and compact fixed point of the set mapping $F(A):=\bigcup_{j=1}^{J} F_{j}(A)$. The set $X$ is also frequently called the self-similar set associated to this IFS; existence and uniqueness of $X$ was shown in Hut81.

From the IFS introduced in the previous definition, we now build a sequence of graphs which approximates $X$ in a suitable sense.

Definition 1.2. We use $\omega=\omega_{1} \omega_{2} \cdots \omega_{m}$ to denote a word of length $|\omega|=m$ on the symbol alphabet $\{1,2, \ldots, J\}$. This notation is used to denote a composition of the mappings $F_{j}$ via $F_{\omega}=F_{\omega_{1}} \circ F_{\omega_{2}} \circ \cdots \circ F_{\omega_{m}}$. Similarly, $K_{\omega}=F_{\omega}(X)$ refers to a certain $m$-level cell. The collection of all finite words is denoted $W_{*}:=\bigcup_{m}\{1,2, \ldots, J\}^{m}$.

Definition 1.3. Each map $F_{j}$ of the IFS defining $X$ has a unique fixed point $x_{j}$. The boundary of $X$ is the largest subset $V_{0} \subseteq\left\{x_{1}, \ldots, x_{N}\right\}$ satisfying

$$
F_{\omega}(X) \cap F_{\omega^{\prime}}(X) \subseteq F_{\omega}\left(V_{0}\right) \cap F_{\omega^{\prime}}\left(V_{0}\right), \quad \text { for any } \omega \neq \omega^{\prime} \text { with }|\omega|=\left|\omega^{\prime}\right| .
$$


The p.c.f. condition mentioned above means that cells $F_{j}(X)$ intersect only at points of $F_{j}\left(V_{0}\right)$. The boundary of an $m$-cell is $\partial K_{\omega}:=F_{\omega}\left(V_{0}\right)$.

Let $G_{0}$ be the complete graph on $V_{0}$, and inductively define $G_{m}:=F\left(G_{m-1}\right)$. Also, we use the notation $x \sim_{m} y$ to indicate that $x$ and $y$ are $m$-level neighbours, i.e., that there is an edge in $G_{m}$ with endpoints $x$ and $y$. We use $V_{m}=F^{m}\left(V_{0}\right)$ to denote the vertices of $G_{m}$, and $V_{*}:=\bigcup_{m} V_{m}$. The fractal $X$ is the closure of $V_{*}$ with respect to either the Euclidean or resistance metric. A discussion of the resistance metric may be found in [Str06, §1.6] or [Kig01, §2.3].

Now we are able to make precise the sense in which $X$ is the limit of graphs: one may compute the Laplacian (and other analytic objects, including graph energy, resistance distance, etc.) for functions $u: X \rightarrow \mathbb{R}$ by computing it on $G_{m}$ and taking the limit as $m \rightarrow \infty$.

Definition 1.4. We assume the existence of a self-similar (Dirichlet) energy form $\mathcal{E}$ on $X$. That is, for functions $u: X \rightarrow \mathbb{R}$, one has

$$
\mathcal{E}(u)=\sum_{j=1}^{J} r_{j}^{-1} \mathcal{E}\left(u \circ F_{j}\right)
$$

for some choice of renormalization factors $r_{1}, \ldots, r_{J} \in(0,1)$ depending on the IFS. This quadratic form is obtained from the approximating graphs as the appropriately renormalized limit of $\mathcal{E}_{G_{m}}(u):=\mathcal{E}_{G_{m}}(u, u)$, where the $m$-level bilinear form is defined as

$$
\mathcal{E}_{G_{m}}(u, v):=\frac{1}{2} \sum_{\substack{x, y \in V_{m} \\ x \widetilde{m} y}} c_{x y}(u(x)-u(y))(v(x)-v(y))
$$

The constant $c_{x y}=c_{x y}^{(m)}$ refers to the conductance of the edge in $G_{m}$ connecting $x$ to $y$ (with $c_{x y}=0$ if there is no such edge). The dependence of $c_{x y}^{(m)}$ on $m$ is typically suppressed, as $x \sim_{m} y$ for at most one value of $m$ on p.c.f. fractals. The domain of $\mathcal{E}$ is

$$
\operatorname{dom} \mathcal{E}:=\{u: X \rightarrow \mathbb{R}: \mathcal{E}(u)<\infty\}
$$

Definition 1.5. We also assume the existence of a self-similar measure $\mu$,

$$
\mu(A)=\sum_{j=1}^{J} \mu_{j} \mu\left(F_{j}^{-1}(A)\right),
$$

with weights $\mu_{j}$ satisfying $0<\mu_{j}<1$ and $\sum_{j} \mu_{j}=1$, and normalized so that $\mu(X)=1$. With the notation of Definition 1.2, the measure of the $m$-cell $K_{\omega}$ is denoted by $\mu\left(K_{\omega}\right)=\mu_{\omega}:=\mu_{\omega_{1}} \mu_{\omega_{2}} \cdots \mu_{\omega_{m}}$. The standard measure refers to the case $\mu_{j}=\frac{1}{J}$, for each $j$.

Remark 1.6. The renormalization factor $r_{j}$ should be confused neither with the contraction factors $\operatorname{Lip}\left(F_{j}\right)$ of the maps of the IFS nor the weights $\mu_{j}$ of the selfsimilar measure $\mu$. The values of these constants are completely independent.

Also, it should be noted that the existence of a self-similar energy asserted in Definition 1.4 is a strong assumption. While the the self-similar measures of Definition 1.5 always exist [Hut81, the existence of the self-similar energy is a much more delicate question; cf. Sab97. 
Definition 1.7. The Laplacian is defined weakly in terms of the energy form. For $u \in \operatorname{dom} \mathcal{E}$ and $f$ continuous, one says that $u \in \operatorname{dom} \Delta$ with $\Delta u=f$ iff

$$
\mathcal{E}(u, v)=-\int_{X} f v d \mu, \quad \text { for all } v \in \operatorname{dom}_{0} \mathcal{E},
$$

where $\operatorname{dom}_{0} \mathcal{E}$ is the set of functions in $\operatorname{dom} \mathcal{E}$ which vanish on $\partial X=V_{0}$. Note that the Laplacian depends on the choice of measure $\mu$.

More generally, if (1.6) holds with $f \in L^{2}(d \mu)$, then one says that $u \in \operatorname{dom}_{L^{2}} \Delta$. Also, if

$$
\mathcal{E}(u, v)=-\int_{X} v d \mu, \quad \text { for all } v \in \operatorname{dom}_{0} \mathcal{E}
$$

for a finite signed measure $\mu$ with no atoms, then one says that $u \in \operatorname{dom}_{\mathcal{M}} \Delta$.

It follows from (1.3), (1.5) and Definition 1.7 that $\Delta$ satisfies the scaling identity

$$
\Delta\left(u \circ F_{j}\right)=r_{j} \mu_{j}(\Delta u) \circ F_{j},
$$

and the pointwise formula given by the uniform limit

$$
\Delta u(x)=\lim _{m \rightarrow \infty}\left(\int_{X} h_{x}^{(m)} d \mu\right)^{-1} \Delta_{m} u(x), \quad \text { for } x \in V_{*} \backslash V_{0},
$$

where $h_{x}^{(m)}$ is a piecewise harmonic spline satisfying $h_{x}^{(m)}(y)=\delta_{x y}$ for $y \in V_{m}$, and

$$
\Delta_{m} u(x)=\sum_{y \widetilde{m}} c_{x y}(u(y)-u(x)), \quad \text { for } x \in V_{m} .
$$

Roughly speaking, $h_{x}^{(m)}$ is a "tent" function with its peak at $x$ which vanishes outside the $m$-cell containing $x$. See [Str06, $§ 2.1-\S 2.2]$ for details.

Definition 1.8. The normal derivative of a function $u$ is computed at a boundary point $q \in V_{0}$ by

$$
\partial_{\mathrm{n}} u(q):=\lim _{m \rightarrow \infty} \frac{1}{r_{i}^{m}} \sum_{y_{\tilde{m}} q}(u(q)-u(y)), \quad q \in V_{0} .
$$

At a general junction point $x=F_{\omega} q$, the normal derivative is computed with respect to a specific $m$-cell $K_{\omega}$ :

$$
\partial_{\mathrm{n}}^{K_{\omega}} u(x)=\partial_{\mathrm{n}}^{K_{\omega}} u\left(F_{\omega} q\right):=\frac{1}{r_{\omega_{1}} \cdots r_{\omega_{m}}} \partial_{\mathrm{n}}\left(u \circ F_{\omega}\right)(q) .
$$

\subsection{Statement of the main result.}

Theorem 1.9. Assume that $\lambda$ is not a Dirichlet eigenvalue of $\Delta$, and neither is $r_{\omega} \mu_{\omega} \lambda$, for any $\omega \in W_{*}$. For the Laplacian on $X$ with Dirichlet boundary conditions, the resolvent kernel $G^{(\lambda)}$ defined by (1.1) is given by the formula

$$
\begin{aligned}
G^{(\lambda)}(x, y) & =\sum_{\omega \in W_{*}} r_{\omega} \Psi^{\left(r_{\omega} \mu_{\omega} \lambda\right)}\left(F_{\omega}^{-1} x, F_{\omega}^{-1} y\right), \\
\text { where } \Psi^{(\lambda)}(x, y) & :=\sum_{p, q \in V_{1} \backslash V_{0}} G_{p q}^{(\lambda)} \psi_{p}^{(\lambda)}(x) \psi_{q}^{(\lambda)}(y),
\end{aligned}
$$


where convention stipulates $\Psi^{\left(r_{\omega} \mu_{\omega} \lambda\right)}\left(F_{\omega}^{-1} x, F_{\omega}^{-1} y\right)=0$ for $x, y$ not in $F_{\omega} X$. In formula (1.14), $\psi_{p}^{(\lambda)}$ is the solution to the resolvent equation at level 1, i.e.

$$
\begin{cases}(\lambda \mathbb{I}-\Delta) \psi_{p}^{(\lambda)}=0, & \text { on each } K_{j}=F_{j}(X), \\ \psi_{p}^{(\lambda)}(q)=\delta_{p q}, & \text { for } p \in V_{1} \backslash V_{0} \text { and } q \in V_{1},\end{cases}
$$

where $\delta_{p q}$ is the Kronecker delta. The coefficients $G_{p q}^{(\lambda)}$ in (1.14) arise as the entries of the inverse of the matrix $B$ given by

$$
B_{p q}^{(\lambda)}:=\sum_{K_{j} \ni q} \partial_{n}^{K_{j}} \psi_{p}^{(\lambda)}(q), \quad q \in F_{j}\left(V_{0}\right)
$$

where the sum is taken over all 1-cells containing $q$.

This result appears with proof as Theorem 3.12. a similar formula for Neumann boundary conditions appears in Theorem 4.2 .

Remark 1.10. In (1.16) and elsewhere, we use the notation $\sum_{K_{j} \ni q}$ to indicate a sum being taken over the set $\left\{j: q \in K_{j}=F_{j}(X)\right\}$.

The rationale for the definitions (1.13)-(1.16) is best explained by the following heuristic argument and by comparison to Str06, Thm. 2.6.1]. One would like $\Psi^{(\lambda)}$ to be a weak solution to the resolvent equation on a 1-cell $C=F_{i}(X)$, except at the boundary where some Dirac masses may appear. However, this implies that $r_{i} \Psi^{\left(r_{i} \mu_{i} \lambda\right)}\left(F_{i}^{-1} x, F_{i}^{-1} y\right)$ will be a weak solution on the 2-cell $F_{i}(C)$, and in the limit (1.13) gives a solution on the entire fractal. Each term added to the partial sum of (1.13) corresponds to canceling the Dirac masses at the previous stage and introducing new ones at the next; these are wiped away in the limit.

For $\Psi^{(\lambda)}$ to be a weak solution at level 1 , we mean that if $u \in \operatorname{dom} \Delta$ and $u$ vanishes on $\partial X=V_{0}$, then

$$
\int_{X} \Psi^{(\lambda)}(x, y)(\lambda \mathbb{I}-\Delta) u(y) d \mu(y)=\sum_{p \in V_{1} \backslash V_{0}} \psi_{p}^{(\lambda)}(x) u(p) .
$$

With (1.14) as given above, integration by parts and linearity give

$$
\begin{aligned}
\int_{X} \Psi^{(\lambda)}(x, y)(\lambda \mathbb{I}-\Delta) u(y) d \mu(y) \\
\quad=\int_{X}\left[\left(\lambda \mathbb{I}-\Delta_{y}\right) \Psi^{(\lambda)}(x, y)\right] u(y) d \mu(y) \\
=\sum_{p, q \in V_{1} \backslash V_{0}} G_{p q}^{(\lambda)} \psi_{p}^{(\lambda)}(x) \int_{X}\left[(\lambda \mathbb{I}-\Delta) \psi_{q}^{(\lambda)}(y)\right] u(y) d \mu(y)
\end{aligned}
$$

where we used the notation $\Delta_{y}$ to indicate that the operator $\Delta$ is applied with respect to the variable $y$.

Now by (1.15), $\psi_{q}^{(\lambda)}$ satisfies the resolvent equation on the interior of the 1cells, but $-\Delta \psi_{q}^{(\lambda)}$ has Dirac masses at the boundary points with weights $B_{q s}^{(\lambda)}:=$ $\sum_{K_{j} \ni s} \partial_{\mathrm{n}}^{K_{j}} \psi_{q}^{(\lambda)}(s)$. In other words, we have $\Delta \psi_{q}^{(\lambda)}=\lambda \psi_{q}^{(\lambda)}$ except on $V_{1} \backslash V_{0}$, so that $(\lambda \mathbb{I}-\Delta) \psi_{q}^{(\lambda)}(y)=\sum_{s \in V_{1} \backslash V_{0}} B_{q s}^{(\lambda)} \delta_{s}(y)$, where $\delta_{s}$ is the Dirac mass at $s$. 
Therefore, the calculation above may be continued:

$$
\begin{aligned}
\int_{X} \Psi^{(\lambda)}(x, y)(\lambda \mathbb{I}-\Delta) u(y) d \mu(y) & =\sum_{p, q, s \in V_{1} \backslash V_{0}} \psi_{p}^{(\lambda)}(x) G_{p q}^{(\lambda)} B_{q s}^{(\lambda)} \int_{X} \delta_{s}(y) u(y) d \mu(y) \\
& =\sum_{p \in V_{1} \backslash V_{0}} \psi_{p}^{(\lambda)}(x) u(p) .
\end{aligned}
$$

The foregoing computation is the origin and motivation for (1.14)-1.16). A key technical point is the use of a linear combination $u$ of vectors $\psi_{q}^{(\lambda)}$ for which $(\lambda I-\Delta) u$ is a single (weighted) Dirac mass at $p$. From the calculation, it is clear that this hinges on the invertibility of $B$; this is the significance of Lemma 3.7

As mentioned just above, once the solution is obtained on level 1, it may be transferred to a cell $F_{\omega}(X)$ by rescaling appropriately. However, this is not sufficient to allow us to compute $\left(\lambda \mathbb{I}-\Delta_{y}\right) G^{(\lambda)}(x, y)$. Some finesse is required to ensure that these solutions match where these cells intersect, that is, on the boundary points $V_{m+1} \backslash V_{0}$. Some further work is needed; this is carried out in the technical lemmas of 93 .

\section{The RESOlVEnt KeRnel FOR THE Unit InTERVAL}

The unit interval $I=[0,1]$ has a self-similar structure derived from the IFS consisting of $F_{1}(x)=\frac{x}{2}$ and $F_{2}(x)=\frac{x}{2}+\frac{1}{2}$. In this section, we exploit this perspective to derive the resolvent kernel for the Dirichlet Laplacian on $I$ by mimicking the construction of the Green function in [Kig01, §3.5] (see also [Str06, §2.6]). This exposition is intended to make the general case (presented in the next section) easier to digest. We build towards the result stated formally in Proposition 2.1.

Proposition 2.1. Let $\Delta=\frac{d^{2}}{d x^{2}}$ be the Laplacian on the unit interval $I=[0,1]$, taken with Dirichlet boundary conditions. If $\lambda$ is not a Dirichlet eigenvalue of $\Delta$, then the resolvent kernel $G^{(\lambda)}$ in (1.1) is given by

$$
\begin{aligned}
G^{(\lambda)}(x, y) & =\sum_{m=0}^{\infty} \sum_{|\omega|=m} \frac{1}{2^{m}} \Psi^{\left(\lambda / 4^{m}\right)}\left(F_{\omega}^{-1} x, F_{\omega}^{-1} y\right), \\
\text { for } \quad \Psi^{(\lambda)}(x, y) & :=\frac{\sinh \frac{\sqrt{\lambda}}{2}}{2 \sqrt{\lambda} \cosh \frac{\sqrt{\lambda}}{2}} \psi^{(\lambda)}(x) \psi^{(\lambda)}(y), \\
\text { and } \quad \psi^{(\lambda)}(x) & :=\frac{1}{\sinh \frac{\sqrt{\lambda}}{2}} \begin{cases}\sinh \sqrt{\lambda} x, & x \leq \frac{1}{2}, \\
\sinh \sqrt{\lambda}(1-x), & x \geq \frac{1}{2},\end{cases}
\end{aligned}
$$

where convention stipulates $\Psi^{\left(\lambda / 4^{m}\right)}\left(F_{\omega}^{-1} x, F_{\omega}^{-1} y\right)=0$ for $x, y$ not in $F_{\omega} I$.

Remark 2.2 (A preview of the general case). Note that the sum in (2.1) is finite if $x \neq y$ or if $x=y$ is dyadic. More importantly, $\psi^{(\lambda)}=\psi_{1 / 2}^{(\lambda)}$ is the solution to the resolvent equation at level 1, i.e.

$$
\begin{cases}(\lambda \mathbb{I}-\Delta) \psi^{(\lambda)}=0, & \text { on }\left(0, \frac{1}{2}\right) \text { and }\left(\frac{1}{2}, 1\right), \\ \psi^{(\lambda)}(0)=\psi^{(\lambda)}(1)=0, & \text { and } \psi^{(\lambda)}\left(\frac{1}{2}\right)=1 .\end{cases}
$$

In 93 , we develop the resolvent kernel in the general case from these observations.

In keeping with the self-similar spirit of the sequel, we use the term 1-cell in reference to the subintervals $\left[0, \frac{1}{2}\right]$ and $\left[\frac{1}{2}, 1\right]$ in the following proof. 

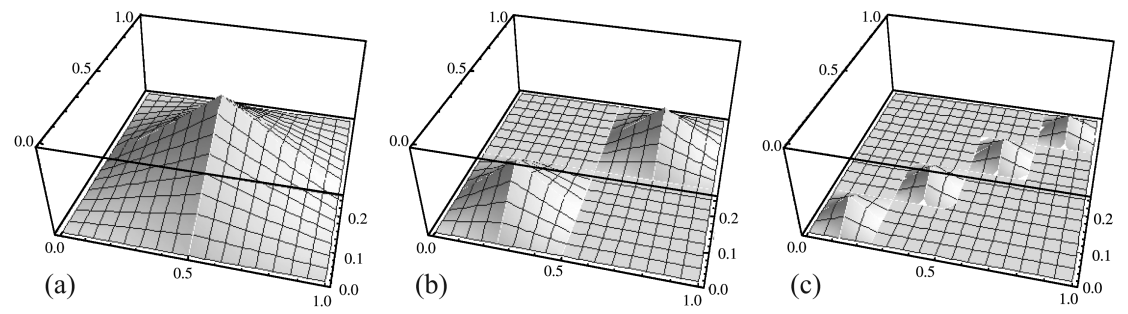

Figure 1. Mathematica plot of $\Psi^{(\lambda)}$ from Proposition 2.1 for $\lambda=$ 1. (a) $\Psi^{(\lambda)}(x, y)$. (b) $\frac{1}{2}\left(\Psi^{(\lambda / 4)}(2 x, 2 y)+\Psi^{(\lambda / 4)}(2 x-1,2 y-1)\right)$. (c) $\frac{1}{4}\left(\Psi^{(\lambda / 16)}(4 x, 4 y)+\Psi^{(\lambda / 16)}(4 x-1,4 y-1)+\Psi^{(\lambda / 16)}(4 x-2,4 y-2)\right.$ $\left.+\Psi^{(\lambda / 16)}(4 x-3,4 y-3)\right)$.
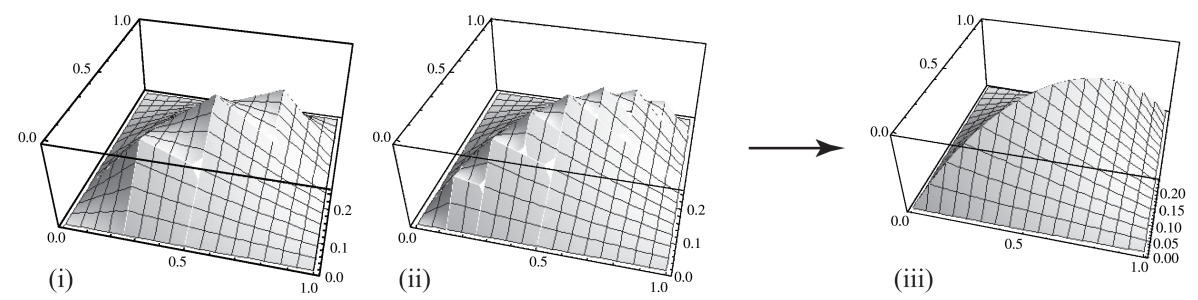

(iii)

Figure 2. Mathematica plot of $G^{(\lambda)}$ for $\lambda=1$ and two of its partial sums. (i) The sum of (a) and (b) in Figure1. (ii) The sum of (a), (b), (c) in Figure 1, (iii) The resolvent kernel $G^{(\lambda)}(x, y)$ obtained in the limit.

Proof of Prop. 2.1. On the unit interval $I$, one has the resolvent kernel

$$
G^{(\lambda)}(x, y)=\frac{1}{\sqrt{\lambda} \sinh \sqrt{\lambda}} \begin{cases}\sinh \sqrt{\lambda}(1-y) \sinh \sqrt{\lambda} x & x \leq y \\ \sinh \sqrt{\lambda} y \sinh \sqrt{\lambda}(1-x), & x \geq y\end{cases}
$$

For $x \leq \frac{1}{2} \leq y$, one has

$$
\begin{array}{rlrl}
G^{(\lambda)}(x, y) & =\frac{\sinh \sqrt{\lambda}(1-y) \sinh \sqrt{\lambda} x}{\sqrt{\lambda} \sinh \sqrt{\lambda}} & \text { by (2.5) } \\
& =\frac{\sinh \frac{\sqrt{\lambda}}{2}}{2 \sqrt{\lambda} \cosh \frac{\sqrt{\lambda}}{2}} \cdot \frac{\sinh \sqrt{\lambda} x \sinh \sqrt{\lambda}(1-y)}{\sinh ^{2} \frac{\sqrt{\lambda}}{2}} & & \sinh 2 a=2 \sinh a \cosh a \\
(2.6) & & =\frac{\sinh \frac{\sqrt{\lambda}}{2}}{2 \sqrt{\lambda} \cosh \frac{\sqrt{\lambda}}{2}} \psi^{(\lambda)}(x) \psi^{(\lambda)}(y) & \text { by (2.3). }
\end{array}
$$

The same computation can be repeated for $y \leq \frac{1}{2} \leq x$, and hence (2.6) holds whenever $x$ and $y$ are in different 1-cells of $I$. 
It remains to consider the case when both $x$ and $y$ lie in the same 1-cell of $I$. Suppose that $x \leq y \leq \frac{1}{2}$ and consider the difference

$$
\begin{aligned}
R(x, y) & :=G^{(\lambda)}(x, y)-\frac{\sinh \frac{\sqrt{\lambda}}{2}}{2 \sqrt{\lambda} \cosh \frac{\sqrt{\lambda}}{2}} \psi^{(\lambda)}(x) \psi^{(\lambda)}(y) \\
& =\frac{\sinh \sqrt{\lambda} x(\sinh \sqrt{\lambda}(1-y)-\sinh \sqrt{\lambda} y)}{\sqrt{\lambda} \sinh \sqrt{\lambda}} \\
& =\frac{\sinh \sqrt{\lambda} x \sinh \sqrt{\lambda}\left(\frac{1}{2}-y\right)}{\sqrt{\lambda} \sinh \frac{\sqrt{\lambda}}{2}} \\
& =\frac{1}{2} G^{(\lambda / 4)}(2 x, 2 y),
\end{aligned}
$$

where (2.7) follows by (2.5) and the identity

$$
\sinh (1-a)-\sinh a=2 \sinh \left(\frac{1}{2}-a\right) \cosh \frac{1}{2} .
$$

In the case when $y \leq x \leq \frac{1}{2}$, one also obtains $R(x, y)=\frac{1}{2} G^{(\lambda / 4)}(2 x, 2 y)$.

On the other hand, when $x$ and $y$ are both in the other 1-cell, one obtains (by analogous computations) that $R(x, y)=\frac{1}{2} G^{(\lambda / 4)}(2 x-1,2 y-1)$. Note that if $\lambda$ is not a Dirichlet eigenvalue of $\Delta$, then neither is $\lambda / 4^{m}$ for any $m=0,1,2, \ldots$. Consequently, if we define $\Psi^{(\lambda)}(x, y)$ as in (2.2), then formula (2.1) for $G^{(\lambda)}(x, y)$ follows.

Remark 2.3. It is interesting to note that the coefficient which appears in (2.6) is

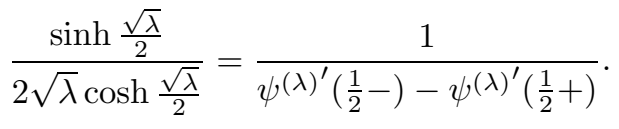

Formally, this indicates $(\lambda \mathbb{I}-\Delta) G^{(\lambda)}(x, y)=\delta(x-y)$; compare to [Str06, (2.6.3)]. Also, observe that

$$
G^{(\lambda)}\left(x, \frac{1}{2}\right)=\frac{\sinh \frac{\sqrt{\lambda}}{2}}{2 \sqrt{\lambda} \cosh \frac{\sqrt{\lambda}}{2}} \psi^{(\lambda)}(x) \psi^{(\lambda)}\left(\frac{1}{2}\right) .
$$

At each successive iteration of (2.8), one is essentially "correcting" the formula on the diagonal for the $m$-cell with rescaled copies of the formula for the $(m+1)$ cell; Figures 11 and 2 are intended to explain this. In the next section, we follow this strategy for the construction of the resolvent kernel in the general case.

Remark 2.4. The procedure in the proof of Proposition 2.1 may also be carried out for the Neumann case: define a function $\varphi^{(\lambda)}$ to be the solution of

$$
\begin{cases}(\lambda \mathbb{I}-\Delta) \varphi^{(\lambda)}=0, & \text { on }\left[0, \frac{1}{2}\right] \text { and }\left[\frac{1}{2}, 1\right] \\ \frac{d}{d x} \varphi^{(\lambda)}(x)=0, & x=0,1 \\ \varphi^{(\lambda)}\left(\frac{1}{2}\right)=1, & \end{cases}
$$

which is given by

$$
\varphi^{(\lambda)}(x)=\frac{1}{\cosh \frac{\sqrt{\lambda}}{2}} \begin{cases}\cosh \sqrt{\lambda} x, & x \leq \frac{1}{2} \\ \cosh \sqrt{\lambda}(1-x), & x \geq \frac{1}{2} .\end{cases}
$$


Observe that in parallel to Remark 2.3 one again has

$$
G_{N}^{(\lambda)}\left(x, \frac{1}{2}\right)=\frac{\cosh \frac{\sqrt{\lambda}}{2}}{2 \sqrt{\lambda} \sinh \frac{\sqrt{\lambda}}{2}} \varphi^{(\lambda)}(x) \varphi^{(\lambda)}\left(\frac{1}{2}\right)
$$

and

$$
\frac{\cosh \frac{\sqrt{\lambda}}{2}}{2 \sqrt{\lambda} \sinh \frac{\sqrt{\lambda}}{2}}=\frac{1}{\frac{d}{d x} \varphi^{(\lambda)}\left(\frac{1}{2}-\right)-\frac{d}{d x} \varphi^{(\lambda)}\left(\frac{1}{2}+\right)} .
$$

By analogous computations, if we define

$$
\Phi_{N}^{(\lambda)}(x, y)=\frac{\cosh \frac{\sqrt{\lambda}}{2}}{2 \sqrt{\lambda} \sinh \frac{\sqrt{\lambda}}{2}} \varphi^{(\lambda)}(x) \varphi^{(\lambda)}(y),
$$

then we obtain the Neumann resolvent kernel

$$
G_{N}^{(\lambda)}(x, y)=\sum_{m=0}^{\infty} \sum_{|\omega|=m} \frac{1}{2^{m}} \Phi^{\left(\lambda / 4^{m}\right)}\left(F_{\omega}^{-1} x, F_{\omega}^{-1} y\right) .
$$

\section{The Dirichlet Resolvent KeRnel For P.C.F. SElF-Similar FraCtals}

In this section, we proceed through a sequence of lemmas which will allow us to prove Theorem 1.9, which is stated in full in Theorem 3.12. On a first reading, the reader may wish to read Theorem 3.12 first, and then work through the lemmas in reverse order. We take one hypothesis of Theorem 1.9 as a blanket assumption throughout this section:

Assumption 3.1. None of the numbers $\lambda_{\omega}=\mu_{\omega} r_{\omega} \lambda$, for $\omega \in W_{*}$, is a Dirichlet eigenvalue of the Laplacian.

We construct the resolvent kernel formula according to the following rough outline:

(1) We build a solution $\eta_{p}^{(\lambda)}$ to the eigenfunction equation which takes the value 1 at one boundary point of $X$ and is 0 on the other boundary points.

(2) We show how $\psi_{p}^{(\lambda)}$ may be written in terms of rescaled copies of $\eta_{p}^{(\lambda)}$, i.e., we decompose the solution around a point $p \in V_{1} \backslash V_{0}$ into solutions for each cell containing $p$.

(3) We use this construction to obtain a solution on the cells of level $m$.

(4) We show how the $(m+1)$-level solution contains Dirac masses on $V_{m}$ which cancel with the Dirac masses of the $m$-level solution, so that the sum over $m$ is telescoping and yields a global solution.

The first two steps are carried out in $\$ 3.1$. In $\$ 3.2$, we collect some properties of $B_{p q}^{(\lambda)}:=\sum_{K_{j} \ni q} \partial_{\mathrm{n}}^{K_{j}} \psi_{p}^{(\lambda)}(q)$, as introduced in 1.15) 1 For each $\lambda$, we think of $B_{p q}^{(\lambda)}$ as the entries of a matrix in $p$ and $q$. Under Assumption 3.1. we show $B^{(\lambda)}$ is symmetric, invertible, and that $\lim _{\lambda \rightarrow 0} B^{(\lambda)}=B^{(0)}$. Finally, the remaining two steps are carried out in $\$ 3.3$

Throughout this section, we will need to analyze the properties of a continuous function that satisfies the $\lambda$-eigenfunction equation on all 1-cells, but whose Laplacian may fail to be in $L^{2}$. Our motivation is that the Laplacian of such a function has Dirac masses at points $p \in V_{1} \backslash V_{0}$ with coefficients that can be computed

\footnotetext{
${ }^{1}$ Recall that $\psi_{p}^{(\lambda)}$ is the solution to the resolvent equation on level 1 as defined in (1.16).
} 
from the normal derivatives. The following result is standard; see [Str06, §2.5], for example.

Proposition 3.2. If $u$ is continuous and $\Delta u=v_{j}$ on each 1-cell $K_{j}=F_{j}(X)$, then $\Delta u$ exists as a measure, as in (1.7), and

$$
\Delta u(x)=\sum_{j=1}^{J} v_{j} \chi_{K_{j}}(x)-\sum_{q \in V_{1} \backslash V_{0}} \delta_{q}(x) \sum_{K_{j} \ni q} \partial_{n}^{K_{j}} u(q),
$$

where $\delta_{q}(x)$ is a Dirac mass and $\partial_{n}^{K_{j}} u(q)$ is the normal derivative of $u$ at $q$ with respect to the cell $K_{j}$, and the sum is expressed in the notation of Remark 1.10.

\subsection{The basic building blocks of the resolvent kernel.}

Lemma 3.3. For any $\lambda$ that is not a Dirichlet eigenvalue of the Laplacian, and for each $p \in V_{0}$, there is a function $\eta_{p}^{(\lambda)}(x) \in \operatorname{dom}_{\mathcal{M}} \Delta$, as in (1.7), which solves

$$
\begin{cases}(\lambda \mathbb{I}-\Delta) \eta_{p}^{(\lambda)}(x)=0, & \text { on } X, \\ \eta_{p}^{(\lambda)}(q)=\delta_{p q}, & \forall q \in V_{0},\end{cases}
$$

where $\delta_{p q}$ is the Kronecker delta. Moreover, if $\zeta_{p}$ is the harmonic function on $X$ with $\zeta_{p}(q)=\delta_{p q}$, then

$$
\begin{aligned}
\eta_{p}^{(\lambda)} & =\zeta_{p}-\lambda \theta_{p}^{(\lambda)} & & \text { on all of } X, \text { and } \\
\partial_{n} \eta_{p}^{(\lambda)}(q) & =\partial_{n} \zeta_{p}(q)-\lambda \kappa_{p q}^{(\lambda)} & & \text { for } q \in V_{0},
\end{aligned}
$$

where $\theta_{p}^{(\lambda)}$ and $\kappa_{p q}^{(\lambda)}$ are meromorphic functions of $\lambda$ with poles at the Dirichlet eigenvalues of the Laplacian, and $\kappa_{p q}^{(\lambda)}=\kappa_{q p}^{(\lambda)}$.

Proof. Let $\left\{f_{n}\right\}$ denote the Dirichlet eigenfunctions of the Laplacian, with the corresponding eigenvalues $\lambda_{n}$ arranged so that $\lambda_{n+1} \geq \lambda_{n}$; equality occurs iff $\lambda_{n}$ has multiplicity greater than one. The functions $f_{n}$ may be assumed orthonormal, and their span is dense in $L^{2}$. Consequently we may write $\zeta_{p}=\sum_{n} a_{p}(n) f_{n}$. The function

$$
\theta_{p}^{(\lambda)}=\sum_{n} \frac{a_{p}(n)}{\lambda-\lambda_{n}} f_{n}
$$

then satisfies

$$
(\lambda \mathbb{I}-\Delta) \theta_{p}^{(\lambda)}=\sum_{n} \frac{a_{p}(n)}{\lambda-\lambda_{n}}\left(\lambda-\lambda_{n}\right) f_{n}=\sum_{n} a_{p}(n) f_{n}=\zeta_{p}
$$

in the $L^{2}$ sense. We also see that $\Delta \theta_{p}^{(\lambda)}=\sum_{n} \lambda_{n} a_{p}(n) f_{n} /\left(\lambda-\lambda_{n}\right)$ is $L^{2}$ convergent, so $\theta_{p}^{(\lambda)} \in \operatorname{dom}_{L^{2}} \Delta$, as in (1.7). In particular, $\theta_{p}^{(\lambda)}$ is continuous and equal to zero on $V_{0}$.

Define $\eta_{p}^{(\lambda)}:=\zeta_{p}-\lambda \theta_{p}^{(\lambda)}$. Then $(\lambda \mathbb{I}-\Delta) \eta_{p}^{(\lambda)}=(\lambda \mathbb{I}-\Delta) \zeta_{p}-\lambda \zeta_{p}=0$, and for $q \in V_{0}$,

$$
\eta_{p}^{(\lambda)}(q)=\zeta_{p}(q)=\delta_{p q}
$$

To verify (3.4), we will need the fact that

$$
\partial_{\mathrm{n}} \eta_{p}^{(\lambda)}(q)=\partial_{\mathrm{n}} \eta_{q}^{(\lambda)}(p)
$$


which follows by computing the normal derivatives as follows:

$$
\begin{array}{rlrl}
\partial_{\mathrm{n}} \eta_{q}^{(\lambda)} & (p)-\partial_{\mathrm{n}} \eta_{p}^{(\lambda)}(q) & \\
& =\sum_{s \in V_{0}}\left(\eta_{p}^{(\lambda)}(s) \partial_{\mathrm{n}} \eta_{q}^{(\lambda)}(s)-\eta_{q}^{(\lambda)}(s) \partial_{\mathrm{n}} \eta_{p}^{(\lambda)}(s)\right) & & \text { by } \\
& =\int_{X}\left(\eta_{p}^{(\lambda)}(x) \Delta \eta_{q}^{(\lambda)}(x)-\eta_{q}^{(\lambda)}(x) \Delta \eta_{p}^{(\lambda)}(x)\right) d \mu(x) & & \text { Gauss-Green } \\
& =0, & & \Delta \eta_{s}^{(\lambda)}=\lambda \eta_{s}^{(\lambda)} .
\end{array}
$$

Now (3.4) follows via

$$
\begin{array}{rlrl}
\partial_{\mathrm{n}} \zeta_{p}(q)-\partial_{\mathrm{n}} \eta_{p}^{(\lambda)}(q) & =\partial_{\mathrm{n}} \zeta_{p}(q)-\partial_{\mathrm{n}} \eta_{q}^{(\lambda)}(p) & & \text { by (3.6) } \\
& =\sum_{s \in V_{0}}\left(\eta_{q}^{(\lambda)}(s) \partial_{\mathrm{n}} \zeta_{p}(s)-\zeta_{p}(s) \partial_{\mathrm{n}} \eta_{q}^{(\lambda)}(s)\right) & & \text { by (3.5) } \\
& =\int_{X}\left(\eta_{q}^{(\lambda)}(x) \Delta \zeta_{p}(x)-\zeta_{p}(x) \Delta \eta_{q}^{(\lambda)}(x)\right) d \mu(x) & & \text { Gauss-Green } \\
& =-\lambda \int_{X} \zeta_{p}(x) \eta_{q}^{(\lambda)}(x) d \mu(x) & & \Delta \zeta_{p}=0 \\
& =\lambda \int_{X}\left(\lambda \zeta_{p}(x) \theta_{q}^{(\lambda)}(x)-\zeta_{p}(x) \zeta_{q}(x)\right) d \mu(x) & & \text { by (3.3) } \\
& =\lambda^{2} \sum_{n} \frac{a_{p}(n) a_{q}(n)}{\lambda-\lambda_{n}}-\lambda \sum_{n} a_{p}(n) a_{q}(n) & & \\
& =\lambda \sum_{n} \frac{\lambda_{n} a_{p}(n) a_{q}(n)}{\lambda-\lambda_{n}} . &
\end{array}
$$

Define for each $p, q \in V_{0}$ the functions

$$
\kappa_{p q}^{(\lambda)}:=\sum_{n} \frac{\lambda_{n} a_{p}(n) a_{q}(n)}{\lambda-\lambda_{n}}
$$

so that $\partial_{\mathrm{n}} \zeta_{q}(p)-\partial_{\mathrm{n}} \eta_{q}^{(\lambda)}(p)=\lambda \kappa_{p q}^{(\lambda)}$. It is evident that $\kappa_{p q}^{(\lambda)}$ is symmetric in $p$ and $q$. It is also meromorphic in $\lambda$ with poles at the points $\lambda_{n}$, as may be verified by writing the expansion on the disc of radius $r$ centered at $z$ (where $z \neq \lambda_{n}$ for any $n$, and $\left.r=\inf _{n}\left|z-\lambda_{n}\right| / 2\right)$ as follows:

$$
\kappa_{p q}^{(\lambda)}=\sum_{n} \frac{\lambda_{n} a_{p}(n) a_{q}(n)}{z-\lambda_{n}} \sum_{k=0}^{\infty}\left(\frac{z-\lambda}{z-\lambda_{n}}\right)^{k}=-\sum_{k=0}^{\infty}(\lambda-z)^{k} \sum_{n} \frac{\lambda_{n} a_{p}(n) a_{q}(n)}{\left(\lambda_{n}-z\right)^{k+1}}
$$

and by using the fact that $\left\{a_{p}(n)\right\}$ and $\left\{a_{q}(n)\right\}$ are in $\ell^{2}$, hence their product is in $\ell^{1}$, while $\lambda_{n} /\left(\lambda_{n}-z\right)^{k+1}$ is bounded for each $k$. Note that $r>0$ because the eigenvalues of a p.c.f. fractal have no finite accumulation point; cf. Kig01, §4.1]. An almost identical argument shows that $\theta_{p}^{(\lambda)}$ is meromorphic in $\lambda$ with values in $\operatorname{dom}_{L^{2}} \Delta$, so the proof is complete.

Corollary 3.4. Let $p \in V_{1} \backslash V_{0}$. If $r_{j} \mu_{j} \lambda$ is not a Dirichlet eigenvalue for any $j$ with $p \in K_{j}$, then $\psi$ and $\eta$ are related via

$$
\psi_{p}^{(\lambda)}(x)= \begin{cases}\eta_{F_{j}^{-1} p}^{\left(r_{j} \mu_{j} \lambda\right)}\left(F_{j}^{-1} x\right) & \text { if } p, x \in K_{j}, \\ 0 & \text { otherwise. }\end{cases}
$$


Proof. From (1.8) we have $\Delta\left(u \circ F_{j}^{-1}\right)=\left(r_{j} \mu_{j}\right)^{-1}(\Delta u) \circ F_{j}^{-1}$, for any $u$. Then from (1.15) and (3.2) one can observe that

$$
\begin{cases}(\lambda \mathbb{I}-\Delta) \eta_{p}^{\left(r_{j} \mu_{j} \lambda\right)} \circ F_{j}^{-1}=0, & \text { on } K_{j}=F_{j}(X), \\ \eta_{p}^{\left(r_{j} \mu_{j} \lambda\right)} \circ F_{j}^{-1}(q)=\delta_{F_{j}(p) q} & \forall q \in F_{j}\left(V_{0}\right) .\end{cases}
$$

Remark 3.5. It is helpful to compare (3.8) to the discussion of the unit interval, where (2.8) may be rewritten as

$$
R(x, y)= \begin{cases}\frac{1}{2} G^{(\lambda / 4)}(2 x, 2 y) & \text { if } x, y \in K_{j} \\ 0 & \text { otherwise }\end{cases}
$$

3.2. The matrix $B^{(\lambda)}$. In the construction of the resolvent kernel, the matrix $B^{(\lambda)}$ plays the same role as the transition matrix for the discrete Laplacian on $V_{1}$ in the corresponding argument of Kigami for the construction of the Dirichlet Green's function. We now collect some important properties of $B^{(\lambda)}$ for use below.

Lemma 3.6. The matrix $B^{(\lambda)}$ is symmetric for any $\lambda$, and $\lim _{\lambda \rightarrow 0} B^{(\lambda)}=B^{(0)}$.

Proof. From (3.6) we have $\partial_{\mathrm{n}}^{K_{j}} \psi_{p}^{(\lambda)}(q)=\partial_{\mathrm{n}}^{K_{j}} \psi_{q}^{(\lambda)}(p)$, and thus $B_{p q}^{(\lambda)}=B_{q p}^{(\lambda)}$. Then from (3.8), if $j_{1}, \ldots, j_{k}$ are those $j$ for which $K_{j}$ contains both $p$ and $q$, then

$$
\begin{aligned}
B_{p q}^{(\lambda)} & =\sum_{i=1}^{k} \partial_{\mathrm{n}}^{K_{j_{i}}}\left(\eta_{F_{j_{i}}^{-1}(p)}^{\left(r_{j_{i}} \mu_{j_{i}} \lambda\right)} \circ F_{j_{i}}^{-1}\right)(q) \\
& =\sum_{i=1}^{k} r_{j_{i}}^{-1} \partial_{\mathrm{n}}^{K_{j_{i}}} \eta_{F_{j_{i}}^{-1}(p)}^{\left(r_{j_{i}} \mu_{j_{i}} \lambda\right)}\left(F_{j_{i}}^{-1}(q)\right) \\
& =\sum_{i=1}^{k} r_{j_{i}}^{-1} \partial_{\mathrm{n}}^{K_{j_{i}}} \zeta_{F_{j_{i}}^{-1}(p)}\left(F_{j_{i}}^{-1}(q)\right)+\sum_{i=1}^{k} r_{j_{i}}^{-1} r_{j_{i}} \mu_{j_{i}} \lambda \kappa_{F_{j_{i}}^{-1}(p) F_{j_{i}}^{-1}(q)}^{\left(r_{j_{j}} \mu_{j_{i}} \lambda\right)} \text { by (3.4) } \\
& =B_{p q}^{(0)}+\lambda \sum_{i=1}^{k} \mu_{j_{i}} \kappa_{F_{j_{i}}^{-1}(p) F_{j_{i}}^{-1}(q)}^{\left(r_{j_{j}} \mu_{j_{i}} \lambda\right)},
\end{aligned}
$$

in which the final sum term is a meromorphic function of $\lambda$ with poles at those $\lambda$ for which $r_{j_{i}} \mu_{j_{i}} \lambda$ is a Dirichlet eigenvalue. We used the observation that the harmonic case with functions $\zeta$ is just the case $\lambda=0$. From (3.9) it is also clear that $B_{p q}^{(\lambda)} \rightarrow B_{p q}^{(0)}$ as $\lambda \rightarrow 0$.

As noted in the discussion following the statement of Theorem 1.9, it is important that the action of $B^{(\lambda)}$ on the subspace $V_{1} \backslash V_{0}$ is invertible.

Lemma 3.7. If $\lambda$ is not a Dirichlet eigenvalue, then $B^{(\lambda)}$ is invertible.

Proof. Suppose that $B^{(\lambda)}=\left[B_{p q}^{(\lambda)}\right]_{p, q \in V_{1} \backslash V_{0}}$ is not invertible, so there are values $a_{q}$ (not all 0) for which $\sum_{q \in V_{1} \backslash V_{0}} B_{p q}^{(\lambda)} a_{q}=0$. Define

$$
u(x):=\sum_{q \in V_{1} \backslash V_{0}} a_{q} \psi_{q}^{(\lambda)}(x) .
$$

It is clear that $(\lambda \mathbb{I}-\Delta) u=0$ on each 1-cell and that $\left.u\right|_{V_{0}}=0$. Now using the notation from Remark 1.10, we compute the sum of the normal derivatives of $u$ 
over cells containing $p$, for any $p \in V_{1} \backslash V_{0}$ :

$$
\begin{aligned}
\sum_{K_{j} \ni p} \partial_{\mathrm{n}}^{K_{j}} u(p) & =\sum_{q \in V_{1} \backslash V_{0}} a_{q} \sum_{K_{j} \ni p} \partial_{\mathrm{n}}^{K_{j}} \psi_{q}^{(\lambda)}(p) \\
& =\sum_{q \in V_{1} \backslash V_{0}} a_{q} B_{q p}^{(\lambda)} \\
& =0,
\end{aligned}
$$

where the last equality follows by applying the symmetry established in Lemma 3.6 to the initial assumption. So Proposition 3.2 implies that $\Delta u$ is continuous. It follows that $(\lambda \mathbb{I}-\Delta) u=0$ on $X$, so $u$ is a Dirichlet eigenfunction with eigenvalue $\lambda$, which is a contradiction.

The next result is used to prove Lemma 3.11 and also makes use of (3.8).

Lemma 3.8. For $p \in V_{1} \backslash V_{0}$ and $q \in V_{0}$ we have

$$
\sum_{s \in V_{1} \backslash V_{0}} B_{p s}^{(\lambda)} \eta_{q}^{(\lambda)}(s)=-B_{p q}^{(\lambda)} .
$$

Proof. For a 1-cell $K_{j}=F_{j}(X)$, the Gauss-Green formula gives

$$
\begin{aligned}
& \sum_{s \in F_{j}\left(V_{0}\right)}\left(\psi_{p}^{(\lambda)}(s) \partial_{\mathrm{n}}^{K_{j}} \eta_{q}^{(\lambda)}(s)-\eta_{q}^{(\lambda)}(s) \partial_{\mathrm{n}}^{K_{j}} \psi_{p}^{(\lambda)}(s)\right) \\
& \quad=\int_{K_{j}}\left(\psi_{p}^{(\lambda)}(x) \Delta \eta_{q}^{(\lambda)}(x)-\eta_{q}^{(\lambda)}(x) \Delta \psi_{p}^{(\lambda)}(x)\right) d \mu(x)=0
\end{aligned}
$$

because both $\psi_{p}^{(\lambda)}(x)$ and $\eta_{q}^{(\lambda)}(x)$ are Laplacian eigenfunctions with eigenvalue $\lambda$ on each 1-cell $K_{j}$. However for $s \in V_{1}$ we have $\psi_{p}^{(\lambda)}(s)=\delta_{p s}$, so this becomes

$$
\partial_{\mathrm{n}}^{K_{j}} \eta_{q}^{(\lambda)}(p)=\sum_{s \in F_{j}\left(V_{0}\right)} \eta_{q}^{(\lambda)}(s) \partial_{\mathrm{n}}^{K_{j}} \psi_{p}^{(\lambda)}(s)
$$

The continuity of the Laplacian of $\eta_{q}^{(\lambda)}$ at $p \in V_{1} \backslash V_{0}$ implies that its normal derivatives sum to zero, as indicated by Proposition 3.2 Thus, summing over 1-cells yields

$$
\begin{aligned}
0=\sum_{j=1}^{J} \partial_{\mathrm{n}}^{K_{j}} \eta_{q}^{(\lambda)}(p) & =\sum_{j=1}^{J} \sum_{s \in F_{j}\left(V_{0}\right)} \eta_{q}^{(\lambda)}(s) \partial_{\mathrm{n}}^{K_{j}} \psi_{p}^{(\lambda)}(s) & & \text { by (3.11) } \\
& =\sum_{s \in V_{1}} \eta_{q}^{(\lambda)}(s) \sum_{K_{j} \ni s} \partial_{\mathrm{n}}^{K_{j}} \psi_{p}^{(\lambda)}(s) & & \text { interchange } \\
& =\sum_{s \in V_{0}} \eta_{q}^{(\lambda)}(s) B_{p s}^{(\lambda)}+\sum_{s \in V_{1} \backslash V_{0}} \eta_{q}^{(\lambda)}(s) B_{p s}^{(\lambda)} & & \text { split } \\
& =B_{p q}^{(\lambda)}+\sum_{s \in V_{1} \backslash V_{0}} B_{p s}^{(\lambda)} \eta_{q}^{(\lambda)}(s) & & \eta_{q}^{(\lambda)}(s)=\delta_{q s} \text { on } V_{0},
\end{aligned}
$$

where we used the sum notation of Remark 1.10 . 
3.3. Construction of the resolvent kernel. Now that we have obtained some necessary properties of $B^{(\lambda)}$, we can proceed with the development of a sequence of technical lemmas required for the proof of the main result. We begin with another corollary of Proposition 3.2 .

Corollary 3.9. If $p \in V_{1}$ and $\lambda$ satisfies Assumption 3.1, then

$$
(\lambda \mathbb{I}-\Delta) \psi_{p}^{(\lambda)}=\sum_{q \in V_{1} \backslash V_{0}} B_{p q}^{(\lambda)} \delta_{q}
$$

Proof. With $\psi_{p}^{(\lambda)}$ and $B_{p q}^{(\lambda)}$ defined as in (1.15)-(1.16), this is clear from (3.1).

Remark 3.10. From the definition in (1.16), we have $B_{p q}^{(\lambda)}=\sum_{K_{j} \ni q} \partial_{\mathrm{n}}^{K_{j}} \psi_{p}^{(\lambda)}(q)$ for $q \in F_{j}\left(V_{0}\right)$. Thus Corollary 3.9 expresses the fact that an application of the resolvent to $\psi_{p}^{(\lambda)}$ leaves behind nothing but a Dirac mass at every point of $V_{1} \backslash V_{0}$, each weighted by the sum of the normal derivatives of $\psi_{p}^{(\lambda)}$.

The conclusion of the following lemma appears very technical, but it expresses a straightforward idea: at each stage $m$, our formula for the resolvent corrects Dirac masses at the $m^{\text {th }}$ level and introduces new ones at the $(m+1)^{\text {th }}$. Thus, summing over $m$ (as we do in Theorem 3.12) produces a telescoping series. This makes precise the comment "these are wiped away in the limit" from the introductory discussion of the main result.

Lemma 3.11. Define $\xi_{p, m}^{(\lambda)}$ to be the unique function solving

$$
\begin{cases}(\Delta-\lambda) \xi_{p, m}^{(\lambda)}=0, & \text { on all } m \text {-cells, } \\ \xi_{p, m}^{(\lambda)}(q)=\delta_{p q}, & \text { for } p \in V_{m} \backslash V_{0} \text { and } q \in V_{m}\end{cases}
$$

Then one has the identity

$$
\begin{aligned}
\left(\lambda \mathbb{I}-\Delta_{y}\right) \sum_{|\omega|=m} r_{\omega} \Psi^{\left(r_{\omega} \mu_{\omega} \lambda\right)}\left(F_{\omega}^{-1} x, F_{\omega}^{-1} y\right) & \\
& =\sum_{p \in V_{m+1} \backslash V_{0}} \xi_{p, m+1}^{(\lambda)}(x) \delta_{p}(y)-\sum_{q \in V_{m} \backslash V_{0}} \xi_{q, m}^{(\lambda)}(x) \delta_{q}(y) .
\end{aligned}
$$

Proof. Since $\Psi^{\left(r_{\omega} \mu_{\omega} \lambda\right)}$ is a sum of functions satisfying the $\lambda$-eigenfunction equation on the level 1 cells $K_{j}$, it is immediate that

$$
\left(\lambda-\Delta_{y}\right) \Psi^{\left(r_{\omega} \mu_{\omega} \lambda\right)}\left(F_{\omega}^{-1} x, F_{\omega}^{-1} y\right)=0, \quad \text { for } y \notin V_{m+1} .
$$

By Proposition 3.2, we therefore need only compute the sum of normal derivatives at points of $V_{m+1}$.

(1) First suppose that $z \in V_{m+1} \backslash V_{m}$ with $z=F_{\omega} p$ for some $|\omega|=m$ and $p \in V_{1} \backslash V_{0}$, so that

$$
\Psi^{\left(r_{\omega} \mu_{\omega} \lambda\right)}\left(F_{\omega}^{-1} x, F_{\omega}^{-1} z\right)=\sum_{s, t \in V_{1} \backslash V_{0}} G_{s t}^{\left(r_{\omega} \mu_{\omega} \lambda\right)} \psi_{s}^{\left(r_{\omega} \mu_{\omega} \lambda\right)}\left(F_{\omega}^{-1} x\right) \psi_{t}^{\left(r_{\omega} \mu_{\omega} \lambda\right)}\left(F_{\omega}^{-1} z\right)
$$


and collecting normal derivatives at $z$ yields

$$
\begin{aligned}
\sum_{F_{\omega}\left(K_{j}\right) \ni z} & \partial_{\mathrm{n}}^{F_{\omega}\left(K_{j}\right)}\left(\Psi^{\left(r_{\omega} \mu_{\omega} \lambda\right)}\left(F_{\omega}^{-1} x, F_{\omega}^{-1} z\right)\right) \\
& =\sum_{s, t \in V_{1} \backslash V_{0}} G_{s t}^{\left(r_{\omega} \mu_{\omega} \lambda\right)} \psi_{s}^{\left(r_{\omega} \mu_{\omega} \lambda\right)}\left(F_{\omega}^{-1} x\right) \sum_{F_{\omega}\left(K_{j}\right) \ni z} \partial_{\mathrm{n}}^{F_{\omega}\left(K_{j}\right)} \psi_{t}^{\left(r_{\omega} \mu_{\omega} \lambda\right)}\left(F_{\omega}^{-1} z\right) \\
& =r_{\omega}^{-1} \sum_{s, t \in V_{1} \backslash V_{0}} G_{s t}^{\left(r_{\omega} \mu_{\omega} \lambda\right)} \psi_{s}^{\left(r_{\omega} \mu_{\omega} \lambda\right)}\left(F_{\omega}^{-1} x\right) B_{t p}^{\left(r_{\omega} \mu_{\omega} \lambda\right)},
\end{aligned}
$$

because

$$
\begin{aligned}
B_{t p}^{\left(r_{\omega} \mu_{\omega} \lambda\right)} & =\sum_{K_{j} \ni p} \partial_{\mathrm{n}}^{K_{j}} \psi_{t}^{\left(r_{\omega} \mu_{\omega} \lambda\right)}(p) & & \text { by (1.16) } \\
& =\sum_{K_{j} \ni F_{\omega}^{-1} z} \partial_{\mathrm{n}}^{K_{j}} \psi_{t}^{\left(r_{\omega} \mu_{\omega} \lambda\right)}\left(F_{\omega}^{-1} z\right) & & p=F_{\omega}^{-1} z \in K_{j} \\
& =r_{\omega} \sum_{F_{\omega}\left(K_{j}\right) \ni z} \partial_{\mathrm{n}}^{F_{\omega}\left(K_{j}\right)}\left(\psi_{t}^{\left(r_{\omega} \mu_{\omega} \lambda\right)} \circ F_{\omega}^{-1}\right)(z), & &
\end{aligned}
$$

where the last line follows from $\partial_{\mathrm{n}}^{K_{\omega}} u\left(F_{\omega}^{-1} q_{i}\right)=r_{\omega} \partial_{\mathrm{n}}\left(u \circ F_{\omega}^{-1}\right)\left(q_{i}\right)$; cf. (1.12).

Continuing the computation from (3.14) and making use of $G:=B^{-1}$, we have

$$
\begin{aligned}
\sum_{F_{\omega}\left(K_{j}\right) \ni z} \partial_{\mathrm{n}}^{F_{\omega}\left(K_{j}\right)}\left(\Psi^{\left(r_{\omega} \mu_{\omega} \lambda\right)}\left(F_{\omega}^{-1} x, F_{\omega}^{-1} z\right)\right) & =r_{\omega}^{-1} \sum_{s \in V_{1} \backslash V_{0}} \delta_{s p}\left(\psi_{s}^{\left(r_{\omega} \mu_{\omega} \lambda\right)}\left(F_{\omega}^{-1}(x)\right)\right) \\
& =r_{\omega}^{-1} \psi_{p}^{\left(r_{\omega} \mu_{\omega} \lambda\right)}\left(F_{\omega}^{-1}(x)\right) \\
& =r_{\omega}^{-1} \xi_{z, m+1}^{(\lambda)}(x),
\end{aligned}
$$

thus showing that $\left(\lambda \mathbb{I}-\Delta_{y}\right)$ has a Dirac mass $\xi_{z, m+1}^{(\lambda)}(x) \delta_{z}(y)$ at $z \in V_{m+1} \backslash V_{m}$.

(2) Next consider a point $z \in V_{m} \backslash V_{0}$. In this case there are several words $\omega_{i}$ for which $z=F_{\omega_{i}}\left(p_{i}\right)$ for some $p_{i} \in V_{0}$. For such a word $\omega$ and such a $p$ we substitute from Lemma 3.8 into (3.14), obtaining

$$
\begin{aligned}
\sum_{K_{j} \ni p} & \partial_{\mathrm{n}}^{F_{\omega}\left(K_{j}\right)}\left(r_{\omega} \Psi^{\left(r_{\omega} \mu_{\omega} \lambda\right)}\left(F_{\omega}^{-1} x, F_{\omega}^{-1} z\right)\right) \\
& =-\sum_{q, s, t \in V_{1} \backslash V_{0}} G_{s, t}^{\left(r_{\omega} \mu_{\omega} \lambda\right)} \psi_{s}^{\left(r_{\omega} \mu_{\omega} \lambda\right)}\left(F_{\omega}^{-1} x\right) B_{t q}^{\left(r_{\omega} \mu_{\omega} \lambda\right)} \eta_{p}^{\left(r_{\omega} \mu_{\omega} \lambda\right)}(q) \\
& =-\sum_{q, s \in V_{1} \backslash V_{0}} \delta_{s q} \psi_{s}^{\left(r_{\omega} \mu_{\omega} \lambda\right)}\left(F_{\omega}^{-1} x\right) \eta_{p}^{\left(r_{\omega} \mu_{\omega} \lambda\right)}(q) \\
& =-\sum_{q \in V_{1} \backslash V_{0}} \psi_{q}^{\left(r_{\omega} \mu_{\omega} \lambda\right)}\left(F_{\omega}^{-1} x\right) \eta_{p}^{\left(r_{\omega} \mu_{\omega} \lambda\right)}(q) .
\end{aligned}
$$

The result is clearly a piecewise $\lambda$-eigenfunction on level $(m+1)$ with respect to the $x$ variable, and so is determined by its values on $V_{m+1}$. In each of the terms (3.15), the values are nonzero only at the points of $V_{m+1}$ that neighbor $z$ in $F_{\omega}(X)$, and they are easily seen to coincide with $\xi_{z, m+1}^{(\lambda)}-\xi_{z, m}^{(\lambda)}$ at these points. Summing over all cells, we conclude that at each $z \in V_{m} \backslash V_{0}$ the operator $(\lambda \mathbb{I}-\Delta)$ has a Dirac $\operatorname{mass}\left(\xi_{z, m+1}^{(\lambda)}-\xi_{z, m}^{(\lambda)}\right) \delta_{z}(y)$, and the result follows. 
Theorem 3.12. Let $\psi_{p}^{(\lambda)}$ be the solution to the resolvent equation at level 1, i.e.

$$
\begin{cases}(\lambda \mathbb{I}-\Delta) \psi_{p}^{(\lambda)}=0, & \text { on each } K_{j}=F_{j}(X), \\ \psi_{p}^{(\lambda)}(q)=\delta_{p q}, & \text { for } p \in V_{1} \backslash V_{0} \text { and } q \in V_{1},\end{cases}
$$

where $\delta_{p q}$ is the Kronecker delta.

Define the kernel

$$
\begin{aligned}
G^{(\lambda)}(x, y) & =\sum_{\omega \in W_{*}} r_{\omega} \Psi^{\left(r_{\omega} \mu_{\omega} \lambda\right)}\left(F_{\omega}^{-1} x, F_{\omega}^{-1} y\right), \\
\text { where } \Psi^{(\lambda)}(x, y) & :=\sum_{p, q \in V_{1} \backslash V_{0}} G_{p q}^{(\lambda)} \psi_{p}^{(\lambda)}(x) \psi_{q}^{(\lambda)}(y) .
\end{aligned}
$$

The coefficients $G_{p q}^{(\lambda)}$ in (3.18) are the entries of the inverse of the matrix $B$ given by

$$
B_{p q}^{(\lambda)}:=\sum_{K_{j} \ni q} \partial_{n}^{K_{j}} \psi_{p}^{(\lambda)}(q), \quad q \in F_{j}\left(V_{0}\right)
$$

the sum taken over all 1-cells containing $q$.

For $\lambda$ satisfying Assumption 3.1, $G^{(\lambda)}(x, y)$ is symmetric and continuous in $x$ and $y$, and is in $\operatorname{dom}_{\mathcal{M}} \Delta_{y}$ with $\left(\lambda \mathbb{I}-\Delta_{y}\right) G^{(\lambda)}(x, y)=\delta_{x}(y)$. As it vanishes on $V_{0}$, it is the Dirichlet resolvent of the Laplacian.

Proof. The symmetry of $G^{(\lambda)}(x, y)$ is obvious. Next, note that

$$
\begin{aligned}
\left(\lambda \mathbb{I}-\Delta_{y}\right) \sum_{m=0}^{M} \sum_{|\omega|=m} r_{\omega} \Psi^{\left(r_{\omega} \mu_{\omega} \lambda\right)}\left(F_{\omega}^{-1} x, F_{\omega}^{-1} y\right) \\
=\sum_{m=0}^{M}\left(\sum_{p \in V_{m+1} \backslash V_{0}} \xi_{p, m+1}^{(\lambda)}(x) \delta_{p}(y)-\sum_{p \in V_{m} \backslash V_{0}} \xi_{p, m}^{(\lambda)}(x) \delta_{p}(y)\right) \\
=\sum_{p \in V_{M+1} \backslash V_{0}} \xi_{p, M+1}^{(\lambda)}(x) \delta_{p}(y)
\end{aligned}
$$

by Lemma 3.11, so that

$$
\lim _{M \rightarrow \infty}\left(\lambda \mathbb{I}-\Delta_{y}\right) \sum_{m=0}^{M} \sum_{|\omega|=m} r_{\omega} \Psi^{\left(r_{\omega} \mu_{\omega} \lambda\right)}\left(F_{\omega}^{-1} x, F_{\omega}^{-1} y\right)=\delta_{x}(y),
$$

in the sense of weak-* convergence. It follows that $G^{(\lambda)}(x, y)$ is in $\operatorname{dom}_{\mathcal{M}}\left(\Delta_{y}\right)$ and that $\left(\lambda \mathbb{I}-\Delta_{y}\right) G^{(\lambda)}(x, y)=\delta_{x}(y)$.

All that remains is to see that $G^{(\lambda)}(x, y)$ is continuous. However, Lemma 3.6 shows $B_{p q}^{\left(r_{\omega} \mu_{\omega} \lambda\right)} \rightarrow B_{p q}^{(0)}$ as $|\omega| \rightarrow \infty$, and hence $G_{p q}^{\left(r_{\omega} \mu_{\omega} \lambda\right)} \rightarrow G_{p q}^{(0)}$. In a similar manner, the relation $\eta_{p}^{\left(r_{\omega} \mu_{\omega} \lambda\right)}=\zeta_{p}+r_{\omega} \mu_{\omega} \lambda \theta_{p}^{\left(r_{\omega} \mu_{\omega} \lambda\right)}$ from Lemma 3.3 shows that $\eta_{p}^{\left(r_{\omega} \mu_{\omega} \lambda\right)} \rightarrow \zeta_{p}$ as $|\omega| \rightarrow \infty$; in particular we find that $\psi_{p}^{\left(r_{\omega} \mu_{\omega} \lambda\right)} \rightarrow \psi_{p}^{(0)}$, and the latter is piecewise harmonic and bounded by 1 . The conclusion is that $\Psi^{\left(r_{\omega} \mu_{\omega} \lambda\right)}$ is bounded as $|\omega| \rightarrow \infty$, and since $r_{\omega}$ is a product of $|\omega|$ terms, all of which are bounded by $\max _{i} r_{i}<1$,

$$
G^{(\lambda)}(x, y)=\sum_{m=0}^{\infty} \sum_{|\omega|=m} r_{\omega} \Psi^{\left(r_{\omega} \mu_{\omega} \lambda\right)}\left(F_{\omega}^{-1} x, F_{\omega}^{-1} y\right)
$$


is bounded by a convergent geometric series. Note that, for each $m$, only a finite number of terms in the second sum are nonzero. As all terms are continuous; so is $G^{(\lambda)}$.

\section{The Neumann Resolvent Kernel for P.C.F. SElf-Similar Fractals}

In Theorem 4.2, we give the formula for the Neumann resolvent kernel.

Lemma 4.1. If $\lambda$ is not a Neumann eigenvalue, then there is $C_{p q}^{(\lambda)}$ such that

$$
\sum_{q \in V_{0}} C_{p q}^{(\lambda)} \partial_{n} \eta_{q}^{(\lambda)}(x)=\delta_{p x}
$$

for $x \in V_{0}$, and $C_{p q}^{(\lambda)}$ is symmetric in $p$ and $q$.

Proof. Since $\lambda$ is not a Neumann eigenvalue, the set of vectors $\left\{\left(\partial_{n} \eta_{q}^{(\lambda)}(x)\right)_{x \in V_{0}}\right\}_{q \in V_{0}}$ is linearly independent, whence the existence of the $C_{p q}^{(\lambda)}$ is immediate. Symmetry follows from (3.6) because the matrix $\left[C_{p q}^{(\lambda)}\right]$ is the inverse of the symmetric matrix $\left[\partial_{n} \eta_{p}^{(\lambda)}(q)\right]$.

From this and Theorem 3.12 we may readily deduce the following result.

Theorem 4.2. If $\lambda$ satisfies Assumption 3.1 and also is not a Neumann eigenvalue, then

$$
G_{N}^{(\lambda)}(x, y)=G^{(\lambda)}(x, y)+\sum_{p, q \in V_{0}} C_{p q}^{(\lambda)} \eta_{p}^{(\lambda)}(x) \eta_{q}^{(\lambda)}(y)
$$

is symmetric, is in $\operatorname{dom}_{\mathcal{M}}\left(\Delta_{y}\right)$, and satisfies $\left(\lambda-\Delta_{y}\right) G_{N}^{(\lambda)}(x, y)=\delta_{x}(y)$ on $X \backslash V_{0}$. It has vanishing normal derivatives on $V_{0}$ and is therefore the Neumann resolvent kernel of the Laplacian.

Proof. The symmetry of $G_{N}^{(\lambda)}(x, y)$ is immediate from the symmetry of $G^{(\lambda)}(x, y)$ and of $C_{p q}^{(\lambda)}$. Both $G^{(\lambda)}(x, y)$ and $\eta_{p}^{(\lambda)}(y)$ are in $\operatorname{dom}_{\mathcal{M}}\left(\Delta_{y}\right)$ and $\left(\lambda-\Delta_{y}\right) \eta_{p}^{(\lambda)}(y)=0$ on $X \backslash V_{0}$, so $\left(\lambda-\Delta_{y}\right) G_{N}^{(\lambda)}(x, y)=\left(\lambda-\Delta_{y}\right) G^{(\lambda)}(x, y)=\delta_{x}(y)$ on $X \backslash V_{0}$.

It remains to prove the assertion about the normal derivatives. We will use the notation $\left(\partial_{\mathrm{n}}\right)_{y} G^{(\lambda)}$ for the normal derivative of $G^{(\lambda)}(x, y)$ with respect to its second variable. Since $G^{(\lambda)}(x, y) \in \operatorname{dom}_{\mathcal{M}}\left(\Delta_{y}\right)$ it has a normal derivative at $p \in V_{0}$, and by the Gauss-Green formula,

$$
\begin{aligned}
\left(\partial_{\mathrm{n}}\right)_{y} G^{(\lambda)}(x, p) & =\sum_{s \in V_{0}}\left(\left(\partial_{\mathrm{n}}\right)_{y} G^{(\lambda)}(x, s) \eta_{p}^{(\lambda)}(s)-G^{(\lambda)}(x, s) \partial_{n} \eta_{p}^{(\lambda)}(s)\right) \\
& =\int_{X}\left(\left(\Delta_{s} G^{(\lambda)}(x, s)\right) \eta_{p}^{(\lambda)}(s)-G^{(\lambda)}(x, s)\left(\Delta_{s} \eta_{p}^{(\lambda)}(s)\right)\right) d \mu(s) \\
& =\int_{X}\left(\Delta_{s}-\lambda\right) G^{(\lambda)}(x, s) \eta_{p}^{(\lambda)}(s) d \mu(s) \\
& =-\eta_{p}^{(\lambda)}(x),
\end{aligned}
$$

where at the first step we used the fact that $G^{(\lambda)}(x, s)=0$ for $s \in V_{0}$ and at the last step we used $\left(\Delta_{s}-\lambda\right) G^{(\lambda)}(x, s)=-\delta_{x}(s)$ as a measure. 
It follows that at each $p \in V_{0}$, the normal derivative of (4.1) vanishes:

$$
\begin{aligned}
\left(\partial_{\mathrm{n}}\right)_{y} G_{N}^{(\lambda)}(x, p) & =-\eta_{p}^{(\lambda)}(x)+\left(\partial_{\mathrm{n}}\right)_{y} \sum_{q, s \in V_{0}} C_{q s}^{(\lambda)} \eta_{q}^{(\lambda)}(x) \eta_{s}^{(\lambda)}(p) & & \text { by (4.2) } \\
& =-\eta_{p}^{(\lambda)}(x)+\sum_{q \in V_{0}} \delta_{q x} \eta_{q}^{(\lambda)}(x) & & \text { by Lemma 4.1 } \\
& =0 . & &
\end{aligned}
$$

\section{Example: The Sierpinski gasket $S G$}

Recall the harmonic extension algorithm as described in [Str06, §1.3]: if the values of a function $u$ are specified at the points of $V_{0}$ and written as a vector

$$
\left.u\right|_{V_{0}}=\left[\begin{array}{c}
u\left(p_{0}\right) \\
u\left(p_{1}\right) \\
u\left(p_{2}\right)
\end{array}\right]
$$

then the harmonic extension of $u$ to $F_{i}\left(V_{0}\right)$ (the boundary points of the 1-cell $\left.F_{i}(S G)\right)$ is given by

$$
\left.u\right|_{F_{i} V_{0}}=\left.A_{i} u\right|_{V_{0}}=\left[\begin{array}{l}
u\left(F_{i} p_{0}\right) \\
u\left(F_{i} p_{1}\right) \\
u\left(F_{i} p_{2}\right)
\end{array}\right]
$$

where

$$
A_{0}=\frac{1}{5}\left[\begin{array}{lll}
5 & 0 & 0 \\
2 & 2 & 1 \\
2 & 1 & 2
\end{array}\right], \quad A_{1}=\frac{1}{5}\left[\begin{array}{lll}
2 & 2 & 1 \\
0 & 5 & 0 \\
1 & 2 & 2
\end{array}\right], \quad \text { and } \quad A_{2}=\frac{1}{5}\left[\begin{array}{lll}
2 & 1 & 2 \\
1 & 2 & 2 \\
0 & 0 & 5
\end{array}\right]
$$

are the harmonic extension matrices. In general, $\left.u\right|_{F_{\omega} V_{0}}=\left.A_{\omega} u\right|_{V_{0}}$, where $A_{\omega}=$ $A_{\omega_{m}} \cdots A_{\omega_{1}}$. Thus, the harmonic extension matrices allow one to construct a harmonic function with specified boundary values. Similarly, spectral decimation provides matrices which allow one to construct an eigenfunction with specified boundary values. For example,

$$
A_{0}(\lambda)=\frac{1}{(5-\lambda)(2-\lambda)}\left[\begin{array}{ccc}
(5-\lambda)(2-\lambda) & 0 & 0 \\
(4-\lambda) & (4-\lambda) & 2 \\
(4-\lambda) & 2 & (4-\lambda)
\end{array}\right]
$$

is the analogue of $A_{0}=A_{0}(0)$. By the usual caveats of spectral decimation, these extension matrices can only be used when $\lambda$ is not a (Dirichlet) eigenvalue.

Remark 5.1 (Spectral decimation). A very brief outline of the method of spectral decimation is as follows:

(1) Begin on some level $m=m_{0}$ with $u_{m}$ and $\lambda_{m}$ that satisfy $-\Delta_{m} u_{m}=\lambda_{m} u_{m}$ on $V_{m} \backslash V_{0}$.

(2) Extend $u_{m}$ to a function $u_{m}$ on $V_{m+1} \backslash V_{0}$ by comparing the eigenvalue equations from each level.

(3) Obtain a collection of extension matrices, one for each mapping in the original IFS, and a rational function $\varrho$ which relates the eigenvalues on one level to the eigenvalues on the previous level by $\varrho\left(\lambda_{m}\right)=\lambda_{m-1}$.

(4) Inductively construct a sequence $\left\{\lambda_{m_{o}}, \lambda_{m_{o}+1}, \lambda_{m_{o}+2}, \ldots\right\}$ by choosing $\lambda_{m+1}$ from the set $\varrho^{-1}\left(\lambda_{m}\right)$ for each $m$. 
For every such sequence that converges, $\alpha \lim _{m \rightarrow \infty} \beta^{m} \lambda_{m}$ will be an eigenvalue of $\Delta$ on $X$, where $\alpha$ and $\beta$ are constants specific to $X$. For the Sierpinski Gasket $S G$, $\alpha=\frac{3}{2}$ and $\beta=3 \frac{5}{3}=5$. Note that the calculations in (2)-(3) will forbid certain choices, so some care must be taken in the construction of $\left\{\lambda_{m}\right\}$. See [Str06, §3] for more details.

To obtain the numbers $B_{p q}^{(\lambda)}$ (appearing in (1.16) ) for the Sierpinski Gasket $S G$, we find the normal derivatives of the eigenfunction that has boundary values $(1,0,0)$, as computed at each point of $V_{0}$. If $(\lambda \mathbb{I}-\Delta) u=0$ but $u$ is not a Dirichlet eigenfunction, then consider $u$ on $F_{0}^{m}\left(V_{0}\right)$. By spectral decimation, this is given by

$$
\left.u\right|_{F_{0}^{m}\left(V_{0}\right)}=\left.A_{0}\left(\lambda_{m}\right) \cdots A_{0}\left(\lambda_{1}\right) u\right|_{V_{0}},
$$

where the matrix $A_{0}(\lambda)$ is as in (5.1). We actually only need the values of the normal derivative

$$
\partial_{\mathrm{n}} u\left(q_{i}\right)=\lim _{m \rightarrow \infty}\left(\frac{5}{3}\right)^{m}\left(2 u\left(q_{i}\right)-u\left(F_{i}^{m} q_{i-1}\right)-u\left(F_{i}^{m} q_{i+1}\right)\right), \quad q_{i} \in V_{0} .
$$

The factor $\frac{5}{3}$ arises here because $r_{j}=\frac{3}{5}$ for each $j$ in (1.3) for $S G$; see also (1.12). The calculation of $r_{j}=\frac{3}{5}$ is given in $\left.\underline{\mathrm{Str} 06}, \S 1.3\right]$.

It is extremely easy to compute the normal derivatives of a harmonic function: one does not need to compute the limit, as all terms of the sequence are equal; see Str06, (2.3.9)]. Therefore, our approach is to obtain a harmonic function which coincides with $u$ on $F_{0}^{m}\left(V_{0}\right)$. The limit of the normal derivatives of these harmonic functions will be the normal derivative of $u$. An alternative interpretation would be to interpret the harmonic functions on $S G$ as the analogue of the linear functions on $I$. Consequently, the tangent to a point of $S G$ should be given by a harmonic function plus a constant, provided the tangent exists. This is the motivating idea of [DRS09.

Multiplication by $A_{0}^{-m}$ allows one to find the required harmonic function at stage $m$; rewriting the normal derivative (5.2) in vector notation, one has

$$
\begin{aligned}
\left.\left(\frac{5}{3}\right)^{m}(2,-1,-1) \cdot u\right|_{F_{0}^{m}\left(V_{0}\right)} & =\left.(2,-1,-1) \cdot A_{0}^{-m} u\right|_{F_{0}^{m}\left(V_{0}\right)} \\
& =\left.(2,-1,-1) \cdot A_{0}^{-m} A_{0}\left(\lambda_{m}\right) \cdots A_{0}\left(\lambda_{1}\right) u\right|_{V_{0}} .
\end{aligned}
$$

It therefore suffices to understand the $\operatorname{limit}_{\lim } A_{0}^{-m} A_{0}\left(\lambda_{m}\right) \cdots A_{0}\left(\lambda_{1}\right)$; this was computed in DRS09. The following theorem is the main result of [DRS09, taken with $m_{0}=0$.

Theorem 5.2. Let $\alpha=(0,1,1)^{T}, \beta=(0,1,-1)^{T}, \gamma_{m}=\left(4,4-\lambda_{m}, 4-\lambda_{m}\right)^{T}$. If neither of the values 2 or 5 occur in the sequence $\lambda_{m}$, then

$$
\begin{gathered}
\lim _{k \rightarrow \infty} A_{0}^{-k} \cdot A_{0}\left(\lambda_{0+k}\right) \cdots A_{0}\left(\lambda_{0+1}\right) \alpha=\frac{4 \lambda}{3 \cdot 5^{0} \lambda_{0}\left(2-\lambda_{0+1}\right)} \prod_{j=2}^{\infty}\left(1-\frac{\lambda_{0+j}}{3}\right) \alpha, \\
\lim _{k \rightarrow \infty} A_{0}^{-k} \cdot A_{0}\left(\lambda_{0+k}\right) \cdots A_{0}\left(\lambda_{0+1}\right) \beta=\frac{2 \lambda}{3 \cdot 5^{0} \lambda_{0}} \beta \\
\lim _{k \rightarrow \infty} A_{0}^{-k} \cdot A_{0}\left(\lambda_{0+k}\right) \cdots A_{0}\left(\lambda_{0+1}\right) \gamma_{0}=(4,4,4)^{T} .
\end{gathered}
$$


In particular, this can be used to get the desired normal derivative. We know that all we need to do is compute

$$
\left.(2,-1,-1) \cdot\left(\lim _{m} A_{0}^{-m} A_{0}\left(\lambda_{m}\right) \cdots A_{0}\left(\lambda_{1}\right)\right) u\right|_{V_{0}} .
$$

The boundary data $\left.u\right|_{V_{0}}$ is taken to be $(1,0,0)$ when computing the normal derivative at the point $p$ where $u(p)=1$, and $(0,1,0)$ at a point where $u(p)=0$ (these two points are the same by symmetry). Writing

$$
\left[\begin{array}{l}
1 \\
0 \\
0
\end{array}\right]=\frac{1}{4}\left[\begin{array}{c}
4 \\
4-\lambda_{0} \\
4-\lambda_{0}
\end{array}\right]-\frac{4-\lambda_{0}}{4}\left[\begin{array}{l}
0 \\
1 \\
1
\end{array}\right] \text { and }\left[\begin{array}{l}
0 \\
1 \\
0
\end{array}\right]=\frac{1}{2}\left[\begin{array}{l}
0 \\
1 \\
1
\end{array}\right]+\frac{1}{2}\left[\begin{array}{c}
0 \\
1 \\
-1
\end{array}\right] \text {, }
$$

we find that

$$
\begin{aligned}
& \lim _{m} A_{0}^{-m} A_{0}\left(\lambda_{m}\right) \cdots A_{0}\left(\lambda_{1}\right)\left[\begin{array}{l}
1 \\
0 \\
0
\end{array}\right] \\
&=\left[\begin{array}{l}
1 \\
1 \\
1
\end{array}\right]-\frac{4-\lambda_{0}}{4} \frac{4 \lambda}{3 \lambda_{0}\left(2-\lambda_{1}\right)} \prod_{j=2}^{\infty}\left(1-\frac{\lambda_{j}}{3}\right)\left[\begin{array}{l}
0 \\
1 \\
1
\end{array}\right],
\end{aligned}
$$

and by (5.3), the normal derivative is

$$
\frac{2\left(4-\lambda_{0}\right) \lambda}{3 \lambda_{0}\left(2-\lambda_{1}\right)} \prod_{j=2}^{\infty}\left(1-\frac{\lambda_{j}}{3}\right)
$$

The normal derivative at the other point is computed by first finding

$$
\begin{aligned}
\lim _{m} A_{0}^{-m} A_{0}\left(\lambda_{m}\right) & \cdots A_{0}\left(\lambda_{1}\right)\left[\begin{array}{l}
0 \\
1 \\
0
\end{array}\right] \\
& =\frac{1}{2} \frac{4 \lambda}{3 \lambda_{0}\left(2-\lambda_{1}\right)} \prod_{j=2}^{\infty}\left(1-\frac{\lambda_{j}}{3}\right)\left[\begin{array}{l}
0 \\
1 \\
1
\end{array}\right]+\frac{1}{2} \frac{2 \lambda}{3 \lambda_{0}}\left[\begin{array}{c}
0 \\
1 \\
-1
\end{array}\right]
\end{aligned}
$$

and then taking the inner product with $(2,-1,-1)$, which cancels the second vector to leave

$$
\frac{-4 \lambda}{3 \lambda_{0}\left(2-\lambda_{1}\right)} \prod_{j=2}^{\infty}\left(1-\frac{\lambda_{j}}{3}\right)
$$

It seems logical at this point to define a function

$$
\tau(\lambda)=\frac{4 \lambda}{3 \lambda_{0}\left(2-\lambda_{1}\right)} \prod_{j=2}^{\infty}\left(1-\frac{\lambda_{j}}{3}\right)
$$

and to write the normal derivative at the point where the 1 occurs as $\left(4-\lambda_{0}\right) \tau(\lambda) / 2$ and at the point where the 0 occurs as $-\tau(\lambda)$. We note that for a non-Dirichlet eigenfunction, none of the values 2, 5,6 occur in $\lambda_{m}$ for $m \geq 1$, so the term $\left(2-\lambda_{1}\right)$ in the denominator cannot be zero. It follows that 3 does not occur for $m \geq 2$ and therefore that $\tau(\lambda) \neq 0$ in this case. An exception to our formula as currently written occurs when $\lambda=0$, because then also $\lambda_{0}=0$, but the function $\tau(\lambda)$ is easily shown to have a continuous extension to $\lambda=0$ with $\tau(0)=1$; cf. DRS09. With this correction, our formula is also valid for the harmonic case. 


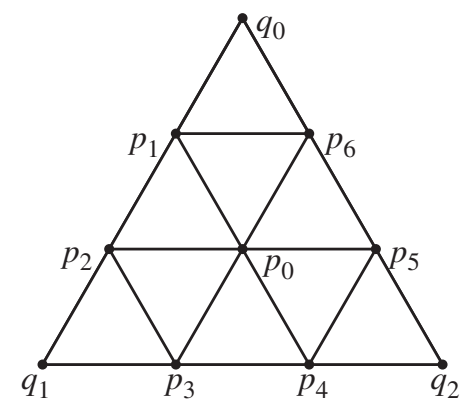

Figure 3. The 1-cells of $S G_{3}$.

It is now easy to write the entries of the matrix $B_{p q}^{(\lambda)}$ appearing in (1.16). The term $B_{p p}^{(\lambda)}$ has two copies of the normal derivative $\left(4-\lambda_{0}\right) \tau(\lambda) / 2$, and the term $B_{p q}^{(\lambda)}$ has a single copy of $-\tau(\lambda)$ at each $q \in V_{1}$ that is not equal to $p$. Both are on 1-cells rather than the whole of $S G$, so there is an extra factor $5 / 3$ in their normal derivatives. As a result, the matrix is

$$
B=\frac{5}{3}\left[\begin{array}{ccc}
\left(4-\lambda_{0}\right) \tau(\lambda) & -\tau(\lambda) & -\tau(\lambda) \\
-\tau(\lambda) & \left(4-\lambda_{0}\right) \tau(\lambda) & -\tau(\lambda) \\
-\tau(\lambda) & -\tau(\lambda) & \left(4-\lambda_{0}\right) \tau(\lambda)
\end{array}\right],
$$

and we should invert this to get the matrix $G_{p q}$ for the Green's function. Since

$$
\operatorname{det}\left[\begin{array}{ccc}
a & b & b \\
b & a & b \\
b & b & a
\end{array}\right]=(a-b)^{2}(a+2 b)
$$

the matrix $B$ is invertible iff $\lambda_{0} \neq 2,5$, in which case

$$
G^{(\lambda)}=\frac{3}{5\left(5-\lambda_{0}\right)\left(2-\lambda_{0}\right) \tau(\lambda)}\left[\begin{array}{ccc}
\left(3-\lambda_{0}\right) & 1 & 1 \\
1 & \left(3-\lambda_{0}\right) & 1 \\
1 & 1 & \left(3-\lambda_{0}\right)
\end{array}\right] .
$$

Note that this is consistent with the harmonic case where $\lambda_{0}=0$ and $\tau(0)=1$ give factors $9 / 50$ for $G_{p p}^{(\lambda)}$ and $3 / 50$ for $G_{p q}^{(\lambda)}$ with $p \neq q$; see [Str06, (2.6.25)].

\section{Example: $S G_{3}$, a VARIant of the Sierpinski Gasket}

6.1. The Laplacian on $S G_{3}$. The fractal $S G_{3}$ is obtained from an IFS consisting of 6 contraction mappings, each with scaling ratio $\frac{1}{3}$, as indicated in Figure 3 . The details of the spectral decimation method for $S G_{3}$ have been worked out independently in [BCD08, DS07, Zho09, Zho10. Note that $p_{0}$ is contained in three 1-cells of $S G_{3}$, in contrast to each of the other points $p_{i}$ of $V_{1} \backslash V_{0}$, which are contained in two. For this reason, we define the graph Laplacian on $S G_{3}$ as

$$
\Delta_{m} u(x)=\frac{1}{\operatorname{deg}(x)} \sum_{y \sim_{m} x}(u(y)-u(x)),
$$

where $\operatorname{deg}(x)$ is the number of $m$-cells containing $x$. From [Str06, §4.4], we have

$$
\Delta_{\mu} u(x)=\lim _{m \rightarrow \infty} r^{-m}\left(\int_{K} h_{x}^{(m)} d \mu\right)^{-1} \operatorname{deg}(x) \Delta_{m} u(x) .
$$




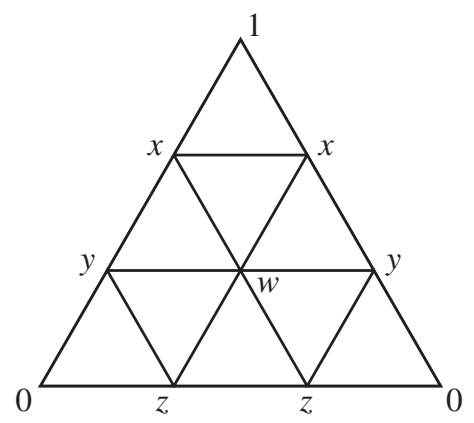

Figure 4. The eigenfunction extension on one $(m-1)$-cell of $S G_{3}$ to $m$-cells. The values on $(m-1)$-cell are $1,0,0$.

The renormalization constant $r$ will be computed in $₫ 6.3$

Let $p_{x}$ denote a vertex where $u$ takes the value $x$ as depicted in Figure 4, Then by (6.1), the symmetric eigenvalue equations on $V_{m}$ are

$$
\begin{aligned}
& \Delta_{m} u\left(p_{x}\right)=\frac{1}{4}[(1-x)+(x-x)+(w-x)+(y-x)]=-\lambda_{m}^{\prime} x, \\
& \Delta_{m} u\left(p_{y}\right)=\frac{1}{4}[(x-y)+(w-y)+(z-y)+(0-y)]=-\lambda_{m}^{\prime} y, \\
& \Delta_{m} u\left(p_{z}\right)=\frac{1}{4}[(y-z)+(w-z)+(z-z)+(0-z)]=-\lambda_{m}^{\prime} z, \\
& \Delta_{m} u\left(p_{w}\right)=\frac{1}{6}[2(x-w)+2(y-w)+2(z-w)]=-\lambda_{m}^{\prime} w,
\end{aligned}
$$

which can be rewritten, using $\lambda_{m}=4 \lambda_{m}^{\prime}$, as

$$
\begin{aligned}
\left(4-\lambda_{m}\right) x & =1+x+y+w, & & \left(4-\lambda_{m}\right) y=x+z+w, \\
\left(4-\lambda_{m}\right) z & =y+z+w, & & \left(4-\lambda_{m}\right) w=\frac{4}{3}(x+y+z) .
\end{aligned}
$$

For now, we suppress the dependence on $m$ for convenience and denote $\lambda=\lambda_{m}$. Solving for $\lambda$, we obtain

$$
\begin{aligned}
x=\alpha(\lambda) & :=\left(96-109 \lambda+33 \lambda^{2}-3 \lambda^{3}\right) / \varphi(\lambda), \\
y=\beta(\lambda) & :=(16-3 \lambda)(3-\lambda) / \varphi(\lambda), \\
z=\gamma(\lambda) & :=(36-7 \lambda) / \varphi(\lambda), \\
w=\rho(\lambda) & :=4(5-\lambda)(3-\lambda) / \varphi(\lambda), \\
\text { where } \quad \varphi(\lambda) & :=3(5-\lambda)(3-\lambda)\left(4-6 \lambda+\lambda^{2}\right),
\end{aligned}
$$

and we see that the forbidden eigenvalues are $3,5,3 \pm \sqrt{5}$.

For a general function on $S G_{3}$, we extend the eigenfunction using the labeling indicated in Figure 5 as follows:

$$
\begin{aligned}
x & =a \alpha(\lambda)+b \beta(\lambda)+c \gamma(\lambda), & & x^{\prime}=a \alpha(\lambda)+c \beta(\lambda)+b \gamma(\lambda), \\
y & =b \alpha(\lambda)+c \beta(\lambda)+a \gamma(\lambda), & & y^{\prime}=b \alpha(\lambda)+a \beta(\lambda)+c \gamma(\lambda), \\
z & =c \alpha(\lambda)+a \beta(\lambda)+b \gamma(\lambda), & & z^{\prime}=c \alpha(\lambda)+b \beta(\lambda)+a \gamma(\lambda), \\
w & =(a+b+c) \rho(\lambda) . & &
\end{aligned}
$$




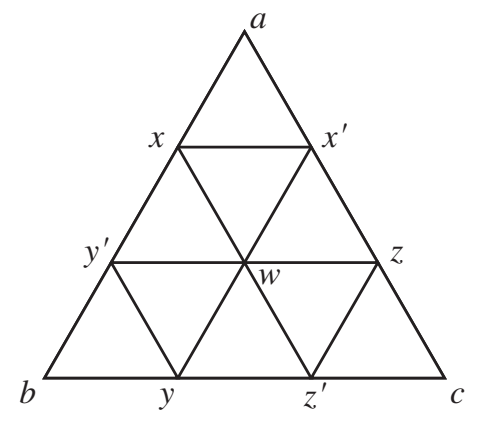

FiguRE 5. The labeling for a general eigenfunction extension, from one $(m-1)$-cell to six $m$-cells. The values on the boundary of the $(m-1)$-cell are $a, b, c$.
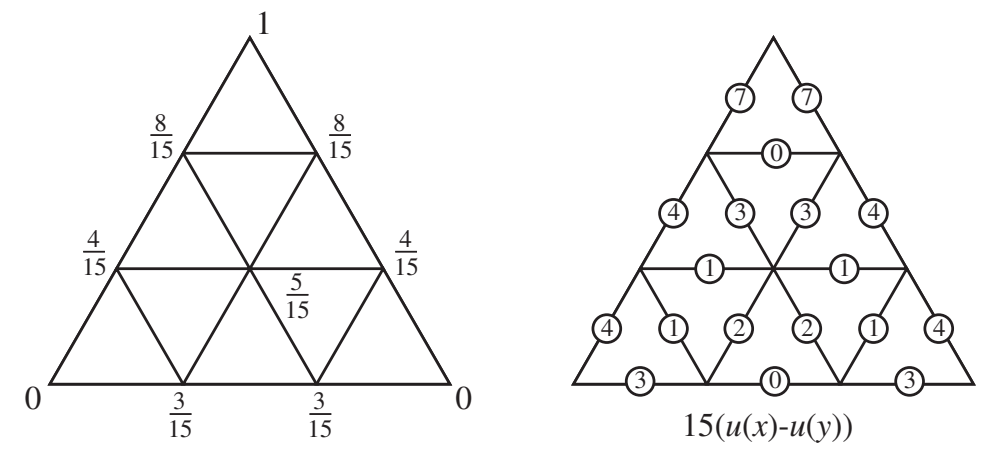

Figure 6. The harmonic extension of $u$ on $S G_{3}$, where $\left.u\right|_{V_{0}}=[1,0,0]$.

The eigenfunction extension matrix for $S G_{3}$ corresponding to $F_{0}$ is

$$
A_{0}(\lambda)=\left[\begin{array}{ccc}
1 & 0 & 0 \\
\alpha(\lambda) & \beta(\lambda) & \gamma(\lambda) \\
\alpha(\lambda) & \gamma(\lambda) & \beta(\lambda)
\end{array}\right]
$$

where we have $\alpha(\lambda), \beta(\lambda), \gamma(\lambda), \varphi(\lambda)$ as before; that is, $\left.A_{0}(\lambda) u\right|_{V_{0}}=\left.u\right|_{F_{0} V_{0}}$.

6.2. Eigenfunctions of the Laplacian on $S G_{3}$. Let $u$ be a function taking values $1,0,0$ on $V_{0}$. The harmonic extension $\tilde{u}$ on $V_{1}$ corresponds to taking $\lambda=0$ in the system (6.3) above, so that

$$
x=\frac{8}{15}, \quad y=\frac{4}{15}, \quad z=\frac{3}{15}, \quad w=\frac{5}{15},
$$

and we have Figure 6. Following [Str06, §1.3], the energy renormalization constant is computed by

$$
E_{1}(\tilde{u})=\left(\frac{1}{15}\right)^{2}\left(4 \cdot 1^{2}+2 \cdot 2^{2}+4 \cdot 3^{2}+4 \cdot 4^{2}+2 \cdot 7^{2}\right)=\frac{14}{15} .
$$

Since $E_{0}(u)=1+1=2$,

$$
2=E_{0}(u)=r^{-1} E_{1}(\tilde{u})=r^{-1} \frac{14}{15} \quad \Longrightarrow \quad r=\frac{7}{15} .
$$




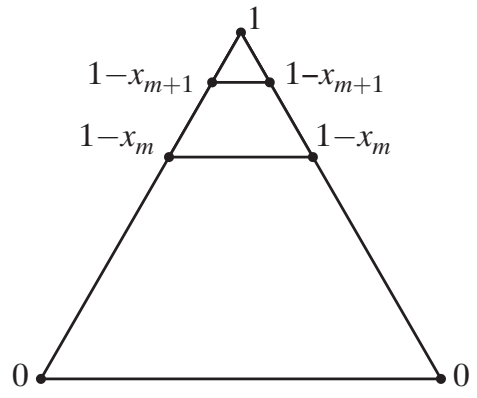

Figure 7 . The values of the eigenfunction $u$ on $S G_{3}$, where $\left.u\right|_{V_{0}}=$ $[1,0,0]^{T}$. This figure shows a closeup of $u$ near the point where it takes the value 1 . By symmetry, we define $x_{m}:=1-u\left(F_{0}^{m} q_{1}\right)=$ $1-u\left(F_{0}^{m} q_{2}\right)$.

Thus, the normal derivatives on $S G_{3}$ are computed by

$$
\partial_{\mathrm{n}} u(p)=\lim _{m \rightarrow \infty}\left(\frac{15}{7}\right)^{m} \sum_{p \sim_{m} y}(u(p)-u(y)) .
$$

Theorem 6.1. The pointwise formulation of the Laplacian on $S G_{3}$ is

$$
\Delta_{\mu} u(x)=6 \lim _{m \rightarrow \infty}\left(\frac{90}{7}\right)^{m} \Delta_{m} u\left(x_{m}\right),
$$

where $\left\{x_{m}\right\}$ is any sequence with $\lim x_{m}=x$ and $x_{m} \in V_{m}$.

Proof. Following [Str06, §2.2], it is easy to compute

$$
\int h_{x_{m}}^{(m)} d \mu=\left\{\begin{array}{cl}
\frac{2}{3 \cdot 6^{m}} & \text { if } \operatorname{deg}\left(x_{m}\right)=4, \\
\frac{1}{6^{m}} & \text { if } \operatorname{deg}\left(x_{m}\right)=6,
\end{array}\right.
$$

since $\mu$ is the standard (self-similar) measure on $S G_{3}$. Thus, by (6.2),

$$
\Delta_{\mu} u(x)=\lim _{m \rightarrow \infty}\left(\frac{15}{7}\right)^{m} \cdot 6^{m+1} \Delta_{m} u(x)=6 \lim _{m \rightarrow \infty}\left(\frac{90}{7}\right)^{m} \Delta_{m} u\left(x_{m}\right) .
$$

Throughout, whenever there is discussion of an eigenvalue $\lambda$, we assume that we have been given the sequence $\left\{\lambda_{m}\right\}_{m=0}^{\infty}$ which defines $\lambda$ via the decimation formula. Thus by Theorem 6.1.

$$
\lambda=6 \lim _{m \rightarrow \infty}\left(\frac{90}{7}\right)^{m} \lambda_{m}^{\prime}=\frac{3}{2} \lim _{m \rightarrow \infty}\left(\frac{90}{7}\right)^{m} \lambda_{m} .
$$

\subsection{Computation of the normal derivatives.}

Theorem 6.2. Let $-\Delta u=\lambda u$ on $S G_{3}$, where $u$ is defined on $V_{0}$ by $u\left(q_{0}\right)=1$, $u\left(q_{1}\right)=0$, and $u\left(q_{2}\right)=0$. Define

$$
\tau(\lambda):=\frac{2 \lambda}{3 \lambda_{0}} \prod_{j=1}^{\infty} \frac{\left(1-\frac{\lambda_{j}}{4}\right)\left(1-\frac{\lambda_{j}}{6}\right)}{1-\frac{3}{2} \lambda_{j}+\frac{\lambda_{j}^{2}}{4}} .
$$


Then the normal derivatives of $u$ are

$$
\begin{aligned}
& \partial_{n} u\left(q_{0}\right)=\frac{4-\lambda_{0}}{2} \tau(\lambda) \quad \text { and } \\
& \partial_{n} u\left(q_{1}\right)=\partial_{n} u\left(q_{2}\right)=-\tau(\lambda) .
\end{aligned}
$$

Proof. To obtain (6.11a) we need the values $u\left(F_{0}^{m} q_{1}\right)=u\left(F_{0}^{m} q_{2}\right)=1-x_{m}$ as depicted in Figure 7 .

Claim. $u\left(F_{0}^{m} q_{1}\right)=u\left(F_{0}^{m} q_{2}\right)=1-x_{m}$, where $x_{0}=1$ and

$$
x_{m+1}-\frac{\lambda_{m+1}}{4}=\frac{\left(4-\lambda_{m+1}\right)\left(6-\lambda_{m+1}\right) \lambda_{m+1}}{\left(4-6 \lambda_{m+1}+\lambda_{m+1}^{2}\right) \lambda_{m}}\left(x_{m}-\frac{\lambda_{m}}{4}\right) .
$$

Proof of the Claim. By (6.4), if $b=c$, then $x=x^{\prime}$. Then from $u\left(q_{1}\right)=u\left(q_{2}\right)=0$, we will have $u\left(F_{0}^{m} q_{1}\right)=u\left(F_{0}^{m} q_{2}\right)$ for all $m$, by induction. Define $x_{m}:=1-u\left(F_{0}^{m} q_{1}\right)$, $m \geq 0$. From $u\left(q_{1}\right)=0$, we have $x_{0}=1$. Now we show that (6.12) holds.

Denote $\delta(\lambda):=\beta(\lambda)+\gamma(\lambda)$ so that $\delta(\lambda)=(14-3 \lambda)(6-\lambda) / \varphi(\lambda)$, where $\varphi(\lambda)$ is as in (6.3). Using (6.4), we have that the matrix equation

$$
A_{0}(\lambda)\left[\begin{array}{c}
1 \\
1-x_{m} \\
1-x_{m}
\end{array}\right]=\left[\begin{array}{c}
1 \\
1-x_{m+1} \\
1-x_{m+1}
\end{array}\right]
$$

gives

$$
\begin{aligned}
x_{m+1} & =1-\alpha\left(\lambda_{m+1}\right)-\delta\left(\lambda_{m+1}\right)+\delta\left(\lambda_{m+1}\right) x_{m} \\
& =-\frac{\lambda_{m+1}\left(5-\lambda_{m+1}\right)}{4-6 \lambda_{m+1}+\lambda_{m+1}^{2}}+\frac{\left(14-3 \lambda_{m+1}\right)\left(6-\lambda_{m+1}\right)}{\varphi\left(\lambda_{m+1}\right)} x_{m} .
\end{aligned}
$$

From the decimation relation DS07, (2.12)], we have the identity

$$
\frac{3\left(5-\lambda_{m+1}\right)\left(4-\lambda_{m+1}\right)\left(3-\lambda_{m+1}\right) \lambda_{m+1}}{\left(14-3 \lambda_{m+1}\right) \lambda_{m}}=1,
$$

so that

$$
\begin{aligned}
\delta\left(\lambda_{m+1}\right) & =\delta\left(\lambda_{m+1}\right) \frac{3\left(5-\lambda_{m+1}\right)\left(4-\lambda_{m+1}\right)\left(3-\lambda_{m+1}\right) \lambda_{m+1}}{\left(14-3 \lambda_{m+1}\right) \lambda_{m}} \\
& =\frac{\left(4-\lambda_{m+1}\right)\left(6-\lambda_{m+1}\right) \lambda_{m+1}}{\left(4-6 \lambda_{m+1}+\lambda_{m+1}^{2}\right) \lambda_{m}} .
\end{aligned}
$$

We would like to see $x_{m+1}-f\left(\lambda_{m+1}\right)=\delta\left(\lambda_{m+1}\right)\left(x_{m}-f\left(\lambda_{m}\right)\right)$ for some function $f$, which is equivalent to

$$
\left(4-6 \lambda_{m+1}+\lambda_{m+1}^{2}\right) \frac{f\left(\lambda_{m+1}\right)}{\lambda_{m+1}}=\frac{f\left(\lambda_{m}\right)}{\lambda_{m}}\left(4-\lambda_{m+1}\right)\left(6-\lambda_{m+1}\right)-\left(5-\lambda_{m+1}\right) .
$$

Let $f(x)=x g(x)$; this can be rewritten as

$$
\left(4-6 \lambda_{m+1}+\lambda_{m+1}^{2}\right) g\left(\lambda_{m+1}\right)=g\left(\lambda_{m}\right)\left(24-10 \lambda_{m+1}+\lambda_{m+1}^{2}\right)-\left(5-\lambda_{m+1}\right),
$$

which is easily seen to be true for the constant function $g(x)=\frac{1}{4}$. Hence we may define $f(x)=\frac{x}{4}$ to obtain

$$
x_{m+1}-\frac{\lambda_{m+1}}{4}=\delta\left(\lambda_{m+1}\right)\left(x_{m}-\frac{\lambda_{m}}{4}\right) .
$$


Now we compute $\partial_{\mathrm{n}} u\left(q_{0}\right)$ using (6.12) to obtain

$$
\begin{aligned}
x_{m}-\frac{\lambda_{m}}{4} & =\left(1-\frac{\lambda_{0}}{4}\right) \frac{\lambda_{m}}{\lambda_{0}} \prod_{j=1}^{m} \frac{\left(4-\lambda_{j}\right)\left(6-\lambda_{j}\right)}{4-6 \lambda_{j}+\lambda_{j}^{2}} \\
x_{m} & =\frac{4-\lambda_{0}}{4 \lambda_{0}}\left(\frac{\lambda_{0}}{4-\lambda_{0}}+\prod_{j=1}^{m} \frac{\left(4-\lambda_{j}\right)\left(6-\lambda_{j}\right)}{4-6 \lambda_{j}+\lambda_{j}^{2}}\right) \lambda_{m} .
\end{aligned}
$$

Since $u\left(q_{0}\right)=1$, we apply (6.6) to compute

$$
\begin{aligned}
\partial_{\mathrm{n}} u\left(q_{0}\right) & =\lim _{m \rightarrow \infty}\left(\frac{15}{7}\right)^{m}\left(\frac{6}{6}\right)^{m}\left(2 u\left(q_{0}\right)-2\left(1-x_{m}\right)\right) \\
& =\frac{4-\lambda_{0}}{2 \lambda_{0}} \lim _{m \rightarrow \infty}\left(\frac{90}{7}\right)^{m} \lambda_{m}\left(\frac{\lambda_{0}}{6^{m}\left(4-\lambda_{0}\right)}+\prod_{j=1}^{m} \frac{\left(4-\lambda_{j}\right)\left(6-\lambda_{j}\right)}{6\left(4-6 \lambda_{j}+\lambda_{j}^{2}\right)}\right) \\
& =\frac{4-\lambda_{0}}{2 \lambda_{0}}\left(\frac{2}{3} \lambda\right)\left(0+\prod_{j=1}^{\infty} \frac{\left(4-\lambda_{j}\right)\left(6-\lambda_{j}\right)}{6\left(4-6 \lambda_{j}+\lambda_{j}^{2}\right)}\right),
\end{aligned}
$$

which is equivalent to the result.

Now we compute the normal derivatives 6.11a . To obtain $\partial_{\mathrm{n}} u\left(q_{1}\right)=\partial_{\mathrm{n}} u\left(q_{2}\right)$, we don't actually need the values $u\left(F_{1}^{m} q_{0}\right)$ and $u\left(F_{1}^{m} q_{2}\right)$ as depicted in Figure 8 . Instead, it suffices to only compute their sum, since by (6.6) one has

$$
\partial_{\mathrm{n}} u\left(q_{1}\right)=\partial_{\mathrm{n}} u\left(q_{2}\right)=-\lim _{m \rightarrow \infty}\left(\frac{15}{7}\right)^{m}\left(u\left(F_{1}^{m} q_{0}\right)+u\left(F_{1}^{m} q_{2}\right)\right) .
$$

To exploit this symmetry accordingly, define

$$
y_{m}:=u\left(F_{0}^{m} q_{1}\right), \quad z_{m}:=u\left(F_{0}^{m} q_{2}\right), \quad \text { and } \quad s_{m}:=y_{m}+z_{m} .
$$

Claim. The sequence $\left\{s_{m}\right\}_{m=0}^{\infty}$ is given recurrently by $s_{0}=1$ and

$$
s_{m+1}=\frac{\left(14-3 \lambda_{m+1}\right)\left(6-\lambda_{m+1}\right)}{\varphi\left(\lambda_{m+1}\right)} s_{m} .
$$

Proof of the Claim. As indicated in Figure 8, dihedral symmetry allows us to continue using the same matrix $A_{0}(\lambda)$ for computations, as long as we use $[0,1,0]^{T}$ for the new boundary data.

It is clear that $s_{0}=1+0$ from the values on $V_{0}$. Then using the notation $\delta(\lambda)=\alpha(\lambda)+\beta(\lambda)$ as above, the matrix equation

$$
A_{0}(\lambda)\left[\begin{array}{c}
0 \\
y_{m} \\
z_{m}
\end{array}\right]=\left[\begin{array}{c}
0 \\
\beta\left(\lambda_{m+1}\right) y_{m}+\gamma\left(\lambda_{m+1}\right) z_{m} \\
\gamma\left(\lambda_{m+1}\right) y_{m}+\beta\left(\lambda_{m+1}\right) z_{m}
\end{array}\right]=\left[\begin{array}{c}
0 \\
y_{m+1} \\
z_{m+1}
\end{array}\right]
$$

gives $s_{m+1}=y_{m+1}+z_{m+1}=\delta\left(\lambda_{m+1}\right) s_{m}$ immediately.

Since (6.14) gives

$$
s_{m}=\prod_{j=1}^{m} \delta\left(\lambda_{j}\right) s_{0}=\frac{\lambda_{m}}{\lambda_{0}} \prod_{j=1}^{m} \frac{\left(4-\lambda_{j}\right)\left(6-\lambda_{j}\right)}{4-6 \lambda_{j}+\lambda_{j}^{2}}
$$



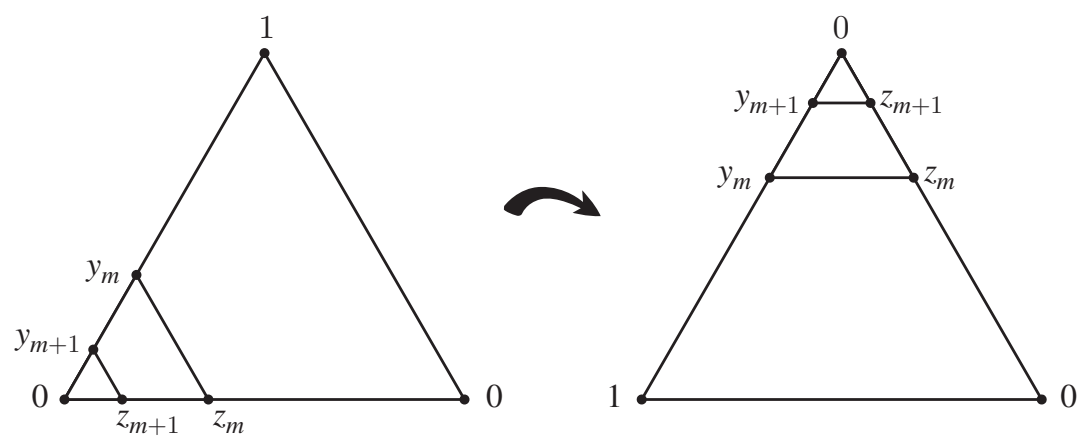

Figure 8. The values of the eigenfunction $u$ on $S G_{3}$, where $\left.u\right|_{V_{0}}=$ $[0,1,0]^{T}$. This figure shows a closeup of $u$ near a point where it takes the value 0 . See (6.13) and the ensuing discussion.

and $u\left(q_{1}\right)=0$, the normal derivative is

$$
\begin{aligned}
\partial_{\mathrm{n}} u\left(q_{1}\right) & =\lim _{m \rightarrow \infty}\left(\frac{15}{7}\right)^{m}\left(\frac{6}{6}\right)^{m}\left(2 u\left(q_{1}\right)-s_{m}\right) \\
& =-\frac{1}{\lambda_{0}} \lim _{m \rightarrow \infty}\left(\frac{90}{7}\right)^{m} \lambda_{m} \prod_{j=1}^{m} \frac{\left(4-\lambda_{j}\right)\left(6-\lambda_{j}\right)}{6\left(4-6 \lambda_{j}+\lambda_{j}^{2}\right)} \\
& =-\frac{1}{\lambda_{0}}\left(\frac{2}{3} \lambda\right) \prod_{j=1}^{\infty} \frac{\left(4-\lambda_{j}\right)\left(6-\lambda_{j}\right)}{6\left(4-6 \lambda_{j}+\lambda_{j}^{2}\right)} .
\end{aligned}
$$

6.4. The resolvent prekernel. As in (1.16), let $B_{p q}^{(\lambda)}:=\sum_{K_{j} \ni q} \partial_{\mathrm{n}}^{K_{j}} \psi_{\lambda}^{(p)}(q)$ for $p \in V_{1} \backslash V_{0}$.

Corollary 6.3. With $\tau(\lambda)$ as in Theorem 6.2 and $r=\frac{7}{15}$,

$$
B_{p q}^{(\lambda)}=-r^{-1} \tau(\lambda), \text { for } p \sim_{1} q, \quad \text { and } \quad B_{p p}^{(\lambda)}= \begin{cases}\frac{3}{2} r^{-1}\left(4-\lambda_{0}\right) \tau(\lambda), & p=p_{0} \\ r^{-1}\left(4-\lambda_{0}\right) \tau(\lambda), & p \neq p_{0} .\end{cases}
$$

Proof. We are now working on $V_{1}$, so each term has a leading factor of $r^{-1}$. Whenever $p \sim_{1} q$, there is just one term $\partial_{\mathrm{n}} u(q)=-\tau(\lambda)$ in the sum. The other corner of the triangle is ignored, and everything outside this 1 -cell is 0 . When $p=q$, there is a sum of terms $\partial_{\mathrm{n}} u(p)=\frac{4-\lambda_{0}}{2} \tau(\lambda)$. At the center point $p_{0}$, there are three such terms; at every other point there are only two.

The matrix $B_{p q}^{(\lambda)}$ is

$$
\frac{15}{7} \tau(\lambda)\left[\begin{array}{ccccccc}
\frac{3}{2}\left(4-\lambda_{0}\right) & -1 & -1 & -1 & -1 & -1 & -1 \\
-1 & \left(4-\lambda_{0}\right) & -1 & 0 & 0 & 0 & -1 \\
-1 & -1 & \left(4-\lambda_{0}\right) & -1 & 0 & 0 & 0 \\
-1 & 0 & -1 & \left(4-\lambda_{0}\right) & -1 & 0 & 0 \\
-1 & 0 & 0 & -1 & \left(4-\lambda_{0}\right) & -1 & 0 \\
-1 & 0 & 0 & 0 & -1 & \left(4-\lambda_{0}\right) & -1 \\
-1 & -1 & 0 & 0 & 0 & -1 & \left(4-\lambda_{0}\right)
\end{array}\right]
$$

Definition 6.4. Define the resolvent prekernel by $G^{(\lambda)}:=\left(B^{(\lambda)}\right)^{-1}$. 
Our final result may be obtained by brutal and direct computation.

Theorem 6.5. The resolvent prekernel $G^{(\lambda)}$ is given by

$$
\frac{14}{15(6-\lambda) \tau(\lambda) \varphi(\lambda)}\left[\begin{array}{ccccccc}
(2-\lambda) \kappa_{1} & \kappa_{1} & \kappa_{1} & \kappa_{1} & \kappa_{1} & \kappa_{1} & \kappa_{1} \\
\kappa_{1} & \kappa_{2} & \kappa_{3} & \kappa_{4} & \kappa_{5} & \kappa_{4} & \kappa_{3} \\
\kappa_{1} & \kappa_{3} & \kappa_{2} & \kappa_{3} & \kappa_{4} & \kappa_{5} & \kappa_{4} \\
\kappa_{1} & \kappa_{4} & \kappa_{3} & \kappa_{2} & \kappa_{3} & \kappa_{4} & \kappa_{5} \\
\kappa_{1} & \kappa_{5} & \kappa_{4} & \kappa_{3} & \kappa_{2} & \kappa_{3} & \kappa_{4} \\
\kappa_{1} & \kappa_{4} & \kappa_{5} & \kappa_{4} & \kappa_{3} & \kappa_{2} & \kappa_{3} \\
\kappa_{1} & \kappa_{3} & \kappa_{4} & \kappa_{5} & \kappa_{4} & \kappa_{3} & \kappa_{2}
\end{array}\right],
$$

where

$$
\begin{aligned}
\kappa_{1} & =(3-\lambda)(5-\lambda)(6-\lambda), \\
\kappa_{2} & =201-300 \lambda+\frac{269}{2} \lambda^{2}-24 \lambda^{3}+\frac{3}{2} \lambda^{4}, \\
\kappa_{3} & =87-75 \lambda+19 \lambda^{2}-\frac{3}{2} \lambda^{3}, \\
\kappa_{4} & =57-24 \lambda+\frac{5}{2} \lambda^{2}, \text { and } \\
\kappa_{5} & =51-15 \lambda-\lambda^{2} .
\end{aligned}
$$

In particular, $G^{(\lambda)}$ is symmetric and invertible with determinant

$$
\operatorname{det} G^{(\lambda)}=\left(\frac{7}{15}\right)^{7} \frac{6\left(4-6 \lambda+\lambda^{2}\right)}{(6-\lambda) \varphi(\lambda)^{2} \tau(\lambda)} .
$$

\section{ACKNOWLEDGEMENTS}

The authors are indebted to the referee for many keen observations and a scrupulously detailed report.

\section{REFERENCES}

[BCD08] Neil Bajorin, Tao Chen, Alon Dagan, Catherine Emmons, Mona Hussein, Michael Khalil, Poorak Mody, Benjamin Steinhurst, and Alexander Teplyaev. Vibration modes of 3n-gaskets and other fractals. J. Phys. A: Math. Theor., 41:015101 (21pp), 2008. MR2450694

[DRS09] Jessica L. DeGrado, Luke G. Rogers, and Robert S. Strichartz. Gradients of Laplacian eigenfunctions on the Sierpinski gasket. Proc. Amer. Math. Soc., (137):531-540, 2009. MR 2448573

[DS07] Sean Drenning and Robert S. Strichartz. Spectral decimation on Hambly's homogeneous hierarchical gaskets. To appear in: Illinois J. Math.

[FHK94] Pat J. Fitzsimmons, Ben M. Hambly, and Takashi Kumagai. Transition density estimates for Brownian motion on affine nested fractals. Comm. Math. Phys., 165(3):595-620, 1994. MR 1301625 (95j:60122)

[HK99] Ben M. Hambly and Takashi Kumagai. Transition density estimates for diffusion processes on post critically finite self-similar fractals. Proc. London Math. Soc. (3), 78(2):431-458, 1999. MR1665249 (99m:60118)

[Hut81] John E. Hutchinson. Fractals and self-similarity. Indiana Univ. Math. J., 30(5):713-747, 1981. MR625600 (82h:49026)

[Kig01] Jun Kigami. Analysis on fractals, volume 143 of Cambridge Tracts in Mathematics. Cambridge University Press, Cambridge, 2001. MR1840042 (2002c:28015)

[Kig03] Jun Kigami. Harmonic analysis for resistance forms. J. Funct. Anal., 204(2):399-444, 2003. MR 2017320 (2004m:31010)

[Rog08] Luke Rogers. Estimates for the resolvent kernel for PCF self-similar fractals. In preparation, 2008. 
[Sab97] Christophe Sabot. Existence and uniqueness of diffusions on finitely ramified self-similar fractals. Ann. Sci. École Norm. Sup. (4), 30(5):605-673, 1997. MR:1474807 (98h:60118)

[See67] Robert T. Seeley. Complex powers of an elliptic operator. In Singular Integrals (Proc. Sympos. Pure Math., Chicago, Ill., 1966), pages 288-307. Amer. Math. Soc., Providence, R.I., 1967. MR0237943 (38:6220)

[See69] Robert T. Seeley. The resolvent of an elliptic boundary problem. Amer. J. Math., 91:889920, 1969. MR0265764 (42:673)

[Str06] Robert S. Strichartz. Differential equations on fractals. Princeton University Press, Princeton, NJ, 2006. A tutorial. MR2246975 (2007f:35003)

[Tep98] Alexander Teplyaev. Spectral analysis on infinite Sierpiński gaskets. J. Funct. Anal., 159(2):537-567, 1998. MR1658094 (99j:35153)

[Zho09] Denglin Zhou. Spectral analysis of Laplacians on the Vicsek set. Pacific J. Math., 241(2):369-398, 2009. MR2507583

[Zho10] Denglin Zhou. Criteria for spectral gaps of Laplacians on fractals. J. Fourier Anal. Appl., 16(1):76-97, 2010.

Department of Mathematics, Cornell University, IthacA, New York 14850-4201

E-mail address: mionescu@math.cornell.edu

Current address: Department of Mathematics, University of Connecticut, Storrs, Connecticut 06269-3009

E-mail address: ionescu@math.unconn.edu

Department of Mathematics, University of Iowa, Iowa City, Iowa 52246-1419

E-mail address: erin-pearse@uiowa.edu

Department of Mathematics, University of Connecticut, Storrs, Connecticut 062693009

E-mail address: rogers@math.uconn.edu

Department of Mathematics, Zhejiang University, Hangzhou, 310027, People's Republic of China - And - Department of Mathematics, Cornell University, Ithaca, New YORK 14850-4201

E-mail address: ruanhj@zju.edu.cn

Department of Mathematics, Cornell University, Ithaca, New York 14850-4201

E-mail address: str@math.cornell.edu 